\title{
Outer Synchronization of Simple Firefly Discrete Models in Coupled Networks
}

\author{
A. Arellano-Delgado, ${ }^{1}$ C. Cruz-Hernández, ${ }^{1}$ \\ R. M. López Gutiérrez, ${ }^{2}$ and C. Posadas-Castillo ${ }^{3}$ \\ ${ }^{1}$ Electronics and Telecommunications Department, Scientific Research and Advanced Studies Center of Ensenada (CICESE), \\ 22860 Ensenada, BC, Mexico \\ ${ }^{2}$ Engineering, Architecture and Design Faculty, Baja California Autonomous University (UABC), 22860 Ensenada, BC, Mexico \\ ${ }^{3}$ Mechanics and Electrical Engineering Faculty, Nuevo León Autonomous University (UANL), 66450 San Nicolás de los Garza, \\ NL, Mexico
}

Correspondence should be addressed to C. Cruz-Hernández; ccruz@cicese.mx

Received 31 December 2014; Accepted 1 May 2015

Academic Editor: Xinkai Chen

Copyright ( 2015 A. Arellano-Delgado et al. This is an open access article distributed under the Creative Commons Attribution License, which permits unrestricted use, distribution, and reproduction in any medium, provided the original work is properly cited.

\begin{abstract}
Synchronization is one of the most important emerging collective behaviors in nature, which results from the interaction in groups of organisms. In this paper, network synchronization of discrete-time dynamical systems is studied. In particular, network synchronization with fireflies oscillators like nodes is achieved by using complex systems theory. Different cases of interest on network synchronization are studied, including for a large number of fireflies oscillators; we consider synchronization in smallworld networks and outer synchronization among different coupled networks topologies; for all presented cases, we provide appropriate ranges of values for coupling strength and extensive numerical simulations are included. In addition, for illustrative purposes, we show the effectiveness of network synchronization by means of experimental implementation of coupled nine electronics fireflies in different topologies.
\end{abstract}

\section{Introduction}

In nature, several complex behaviors emerge from the interaction among living organisms to generate a common ben efit, for example, synchronization in shoaling fish to avoid predators, organization of ants to build the nest, flock of birds, and pod of dolphins, and one of the most spectacular synchronization cases is the firefly flashing in Southeast Asia and North America. In $[1,2]$ some studies on synchronization of fireflies are reported. Such insects use their bioluminescence for mating purposes where thousands of male fireflies flash at the same time in a rhythmic form to attract the attention of nearby females. Synchronization was introduced by C. Huygens when he was surprised with the synchronization of two pendulum clocks coupled mutually by the wall; an experiment of Huygens's clocks is studied in [3]. In the last decades, synchronization has been studied on chemical, biological, and ecological systems and electronics devices [47]. Thus, synchronization is the effect to produce the same dynamic behavior in two or more elements when they are coupled with physical links; in addition, synchronization can be classified into two types, mutual synchronization (bidirectional coupling) and master-slave synchronization (unidirectional coupling); these two types of synchronization are widely discussed in $[8,9]$.

The fireflies are considered as oscillators that are coupled by light pulses. The coupling and synchronization of oscillators have been studied by scientists since the $60 \mathrm{~s}$ in biological systems; see, for example, [10-13]. In 1990, Mirollo and Strogatz published an article where a mathematical model is introduced to couple and synchronize an oscillator by pulses in [14]; they assume that all the oscillators in the system are identical; this model was pioneer to explain the synchronization in complex systems such as neurons, cells 


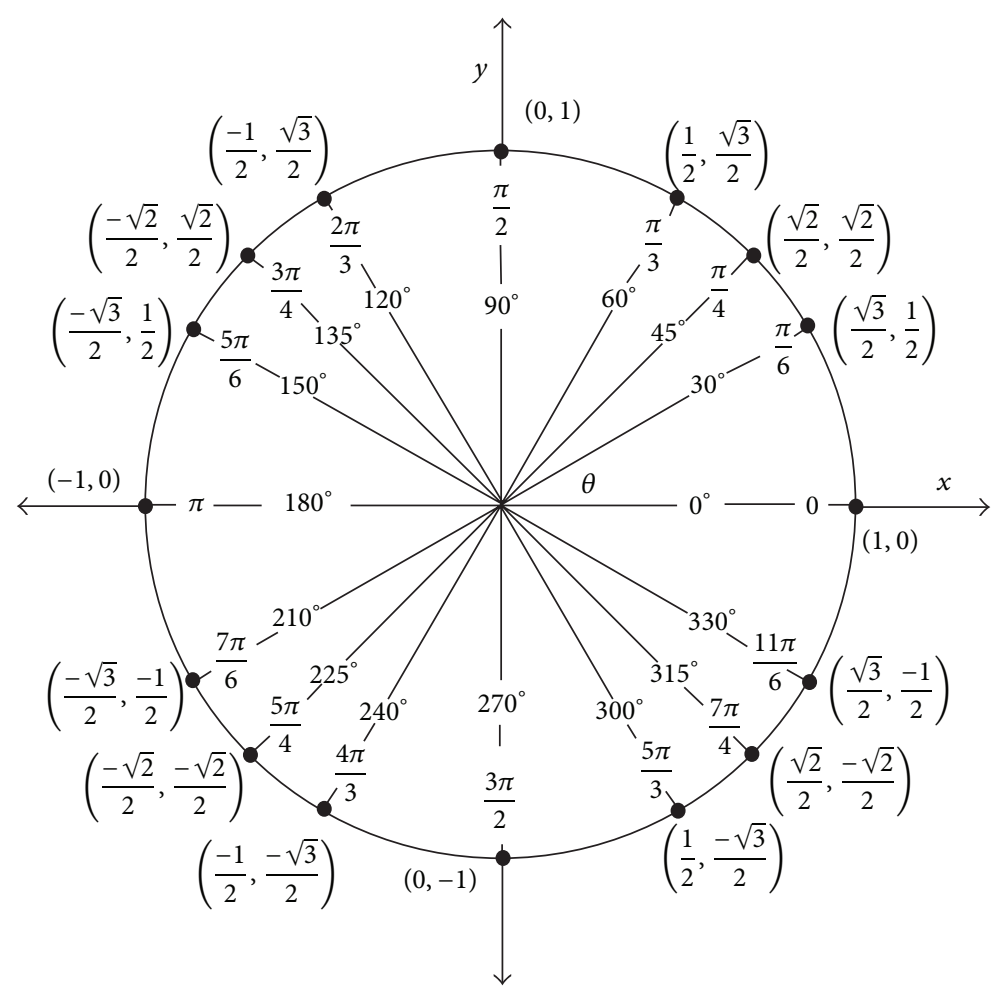

FIGURE 1: The unitary circle that represents a circular unitary motion in the fireflies.

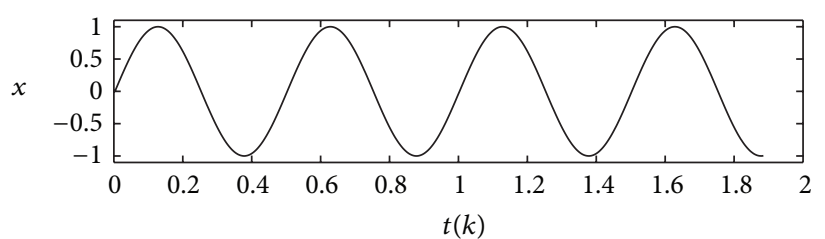

(a)

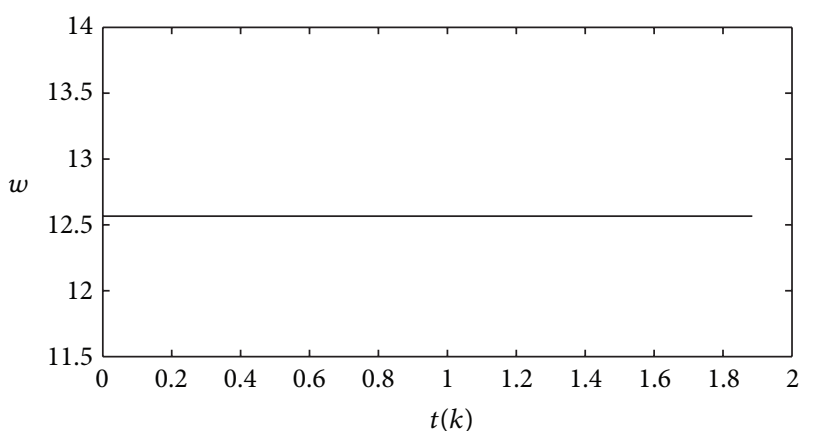

(b)

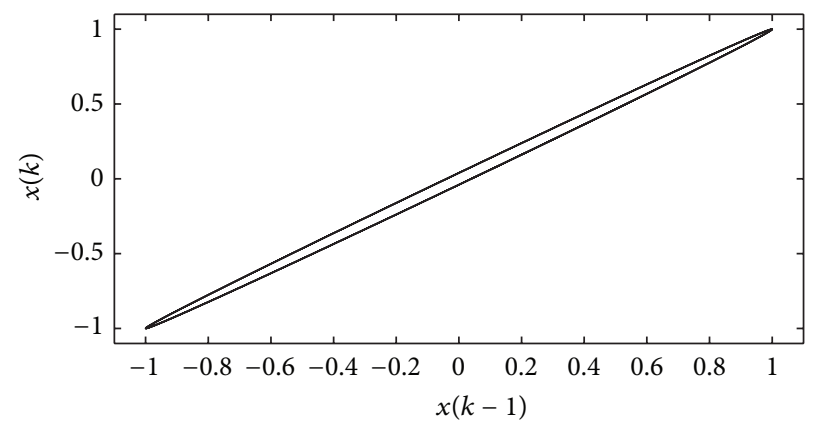

(c)

Figure 2: Behavior of an isolated firefly (7): (a) temporal dynamics $x(k)$ blinking two times per second, (b) angular frequency $w(k)$, and (c) phase-portrait $x(k)$ versus $x(k-1)$. 
of the heart, and fireflies. Another work about the analysis of oscillators coupled by pulses was presented by Ernst et al. in [15] based on the Mirollo-Strogatz model but with coupling cancellation; other approximations to modeling fireflies synchronization have been proposed in $[16,17]$. Therefore, the study and analysis on synchronization of fireflies can help to understand the emergence of collective behavior in other organisms and their applications such as robotics and communications.

In this paper, we use a dynamical model to synchronize in several topologies based on the coupling matrix. First, the model of a firefly is presented and simulated; then, five fireflies are coupled in nearest-neighbor network, star network, and small-world network, where the synchronization is achieved in all the reported cases. We use the MatLab software to simulate the synchronization process that is verified with the graphics of temporal dynamics, phase, and error among the nodes. Additionally, we extend the analysis to outer synchronization of two and three coupled networks; we prove the effectiveness of the proposed synchronization scheme with numerical results and experimental implementation. This paper is organized as follows. In Section 2 we give a brief review on complex dynamical networks. In Section 3, the proposed firefly dynamical discrete simple model is presented. Network synchronization of different topologies is shown in Section 4. In Section 5, the outer synchronization of two and three networks is presented. In Section 6, experimental implementation of fireflies is presented. Finally, some conclusions are mentioned in Section 7.

\section{Preliminaries}

In this section, we give a brief review on synchronization of complex dynamical networks.

2.1. Synchronization of Complex Networks. We consider a complex network is composed of $N$ identical nodes, linearly and diffusively coupled through the first state of each node. In this network, each node constitutes an $N$-dimensional discrete-time map. The state equations of this network are described by

$$
\mathbf{x}_{i}(k+1)=f\left(\mathbf{x}_{i}(k)\right)+\mathbf{u}_{i}(k), \quad i=1,2, \ldots, N
$$

where $\mathbf{x}_{i}(k)=\left(x_{i 1}(k), x_{i 2}(k), \ldots, x_{i N}(k)\right)^{T} \in \mathbb{R}^{N}$ are the state variables of the node $i$ and $\mathbf{u}_{i}(k)=\left(u_{i 1}(k), 0, \ldots, 0\right) \in \mathbb{R}^{N}$ is the input signal of the node $i$ and is defined by

$$
\mathbf{u}_{i}(k)=c \sum_{j=1}^{N} a_{i j} \Gamma \mathbf{x}_{j}(k), \quad i=1,2, \ldots, N,
$$

where the constant $c>0$ represents the coupling strength of the complex network and $\Gamma \in \mathbb{R}^{N \times N}$ is constant $0-1$ matrix linking coupled state variables. Meanwhile, $\mathbf{A}=\left(a_{i j}\right) \in \mathbb{R}^{N \times N}$ is the coupling matrix, which represents the coupling topology of the complex network. If there is a connection between node

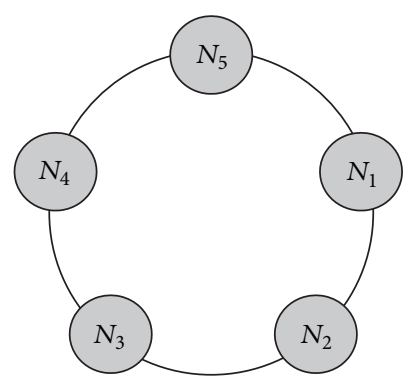

Figure 3: Network of five coupled fireflies in nearest-neighbor topology.

$i$ and node $j$, then $a_{i j}=1$; otherwise, $a_{i j}=0$ for $i \neq j$. The diagonal elements of coupling matrix $\mathbf{A}$ are defined as

$$
a_{i i}=-\sum_{j=1, j \neq i}^{N} a_{i j}=-\sum_{j=1, j \neq i}^{N} a_{j i}, \quad i=1,2, \ldots, N \text {. }
$$

If the degree of node $i$ is $d_{i}$, then $a_{i i}=-d_{i}, i=1,2, \ldots, N$.

Now, suppose that the complex network is connected without isolated clusters. Then, $\mathbf{A}$ is a symmetric irreducible matrix. In this case, it can be shown that zero is an eigenvalue of $\mathbf{A}$ with multiplicity 1 and all the other eigenvalues of $\mathbf{A}$ are strictly negative; see $[18,19]$.

In accordance with [19], the complex network (1)-(2) is said to achieve (asymptotically) synchronization if

$$
\mathbf{x}_{1}(k)=\mathbf{x}_{2}(k)=\cdots=\mathbf{x}_{N}(k), \quad \text { as } k \longrightarrow \infty .
$$

Diffusive coupling condition (3) guarantees that the synchronization state is a solution, $\mathbf{s}(k) \in \mathbb{R}^{N}$, of an isolated node; that is,

$$
\mathbf{s}(k+1)=f(\mathbf{s}(k)),
$$

where $\mathbf{s}(t)$ can be an equilibrium point, a periodic orbit, or a chaotic attractor. Thus, stability of the synchronization state,

$$
\mathbf{x}_{1}(k)=\mathbf{x}_{2}(k)=\cdots=\mathbf{x}_{N}(k)=\mathbf{s}(k)
$$

of complex network (1)-(2) is determined by the dynamics of an isolated node, that is, function $f$ and solution $\mathbf{s}(k)$, the coupling strength $c$, the inner linking matrix $\Gamma$, and the coupling matrix $\mathbf{A}$.

\section{Dynamics of a Simple Firefly Discrete Model}

In this section, a simple firefly discrete-time mathematical model is described, in order to be used as fundamental nodes to construct different coupled networks.

The simple dynamical model of a firefly is expressed by means of the following discrete-time system based on [20]

$$
\begin{aligned}
& x(k+1)=\sin (w(k) t(k)), \\
& t(k+1)=t(k)+\frac{\pi}{1000},
\end{aligned}
$$




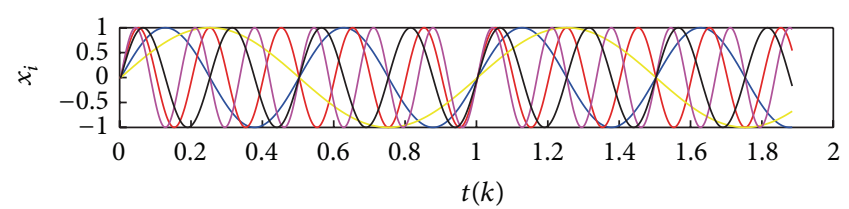

$x_{1}$
$-x_{2}$
$x_{3}$

$$
\begin{gathered}
x_{4} \\
-x_{5}
\end{gathered}
$$

(a)

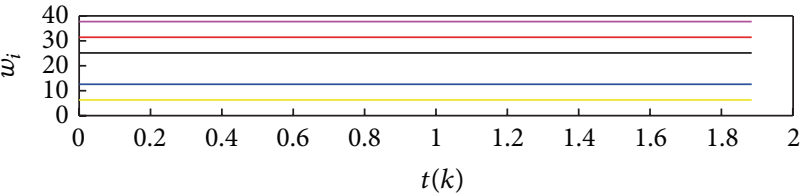

$-w_{1}$
$-w_{2}$
$w_{3}$ $-w_{4}$

(b)

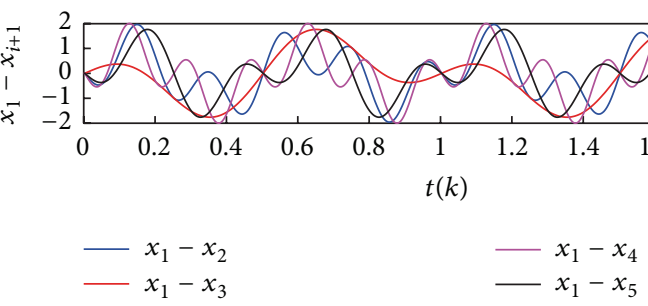

(c)

FIGURE 4: Temporal dynamics of (a) $x_{i}(k)$ and (b) $w_{i}(k)$ with $i=1,2, \ldots, 5$ and error $(\mathrm{c}) x_{1}(k)-x_{i+1}(k)$ with $i=1,2, \ldots, 4$, in the uncoupled nearest-neighbor network.

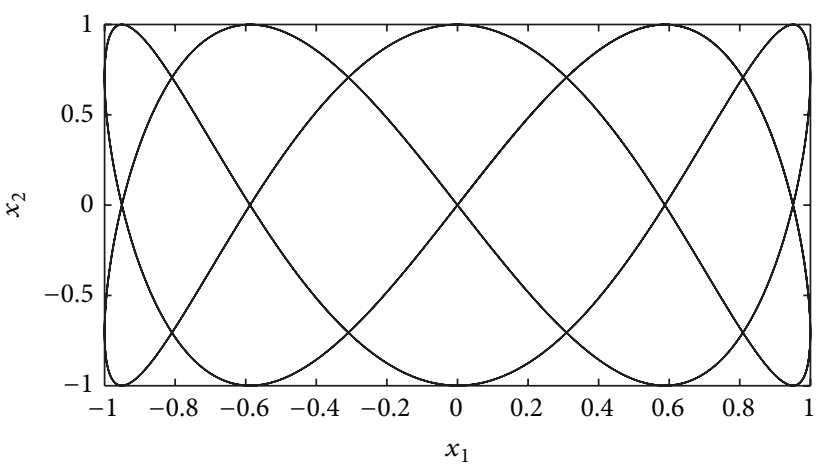

(a)

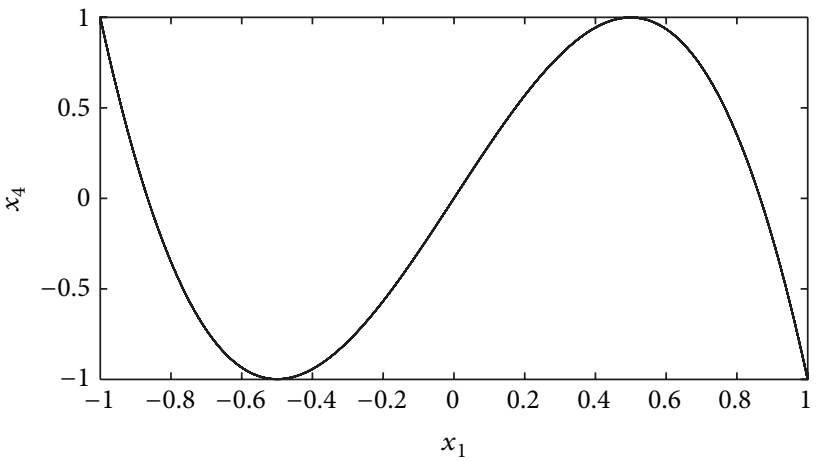

(c)

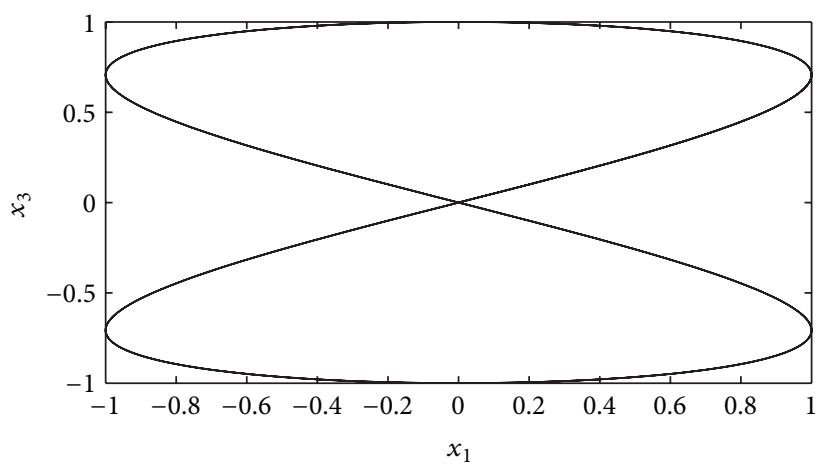

(b)

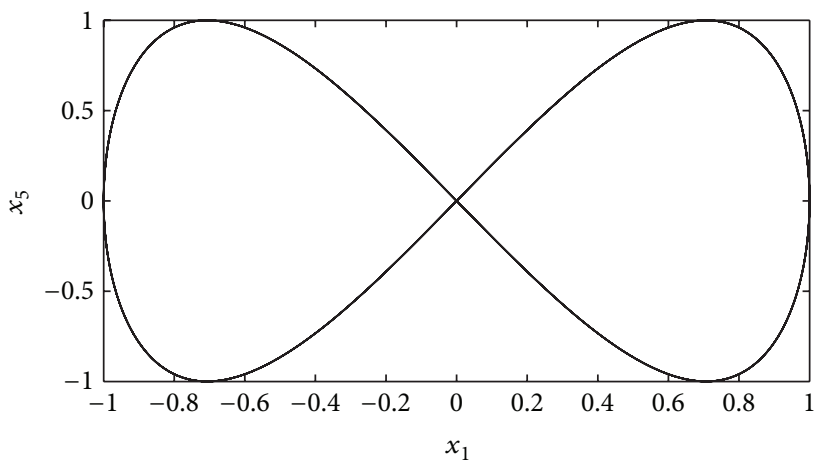

(d)

Figure 5: Phase diagrams (a) $x_{1}$ versus $x_{2}$, (b) $x_{1}$ versus $x_{3}$, (c) $x_{1}$ versus $x_{4}$, and (d) $x_{1}$ versus $x_{5}$ in the uncoupled nearest-neighbor network.

where $x(k)$ is the simple representation of a firefly in discretetime, $w(k)=2 \pi f$ is the angular frequency in radians with $f$ the blink frequency, $t(k)$ is the sampling time, and $k$ is the number of iterations.
Isolated firefly (7) does not receive stimulus from any other firefly in the network, and it is flashing with a period of time of $t$ seconds. If a firefly blinks with a certain period, we take on the sequence of flashes as a circular unitary 


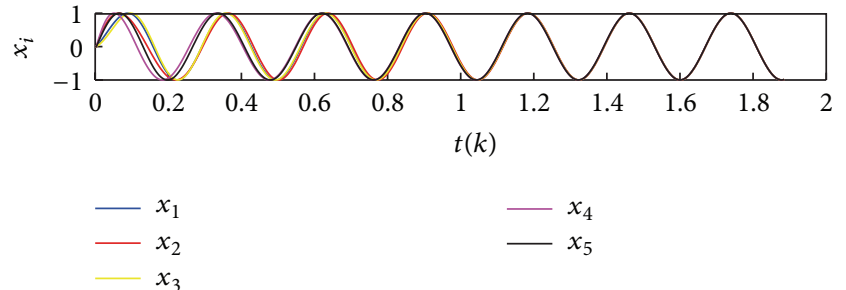

(a)

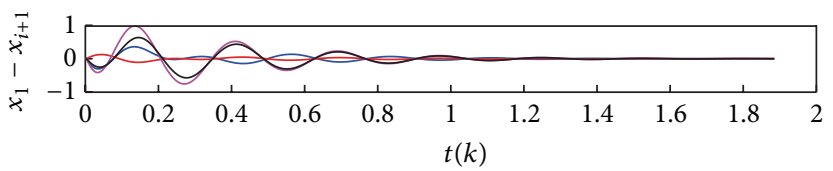

$$
\begin{aligned}
& -x_{1}-x_{2} \\
& -x_{1}-x_{3}
\end{aligned}
$$

(c)

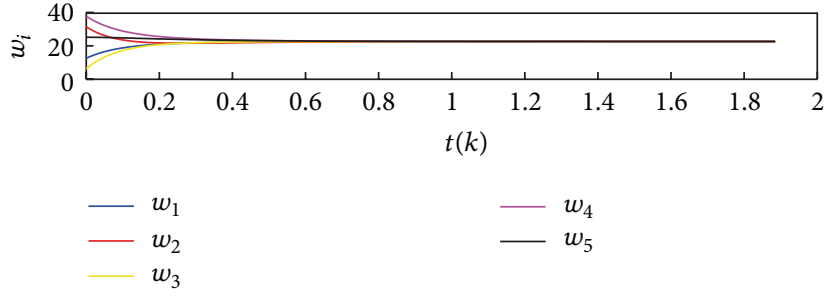

(b)

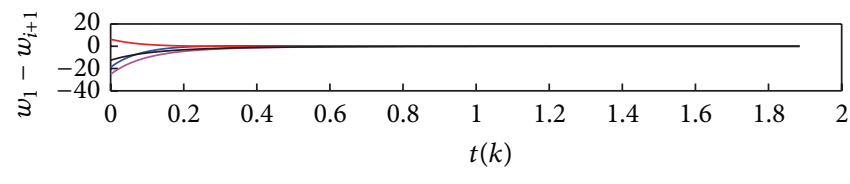

(d)

Figure 6: Temporal dynamics of (a) $x_{i}(k)$ and (b) $w_{i}(k)$ with $i=1,2, \ldots, 5$ and errors (c) $x_{1}(k)-x_{i+1}(k)$ and (d) $w_{1}(k)-w_{i+1}(k)$ with $i=1,2, \ldots, 4$, in the coupled nearest-neighbor network with $c=0.01$.

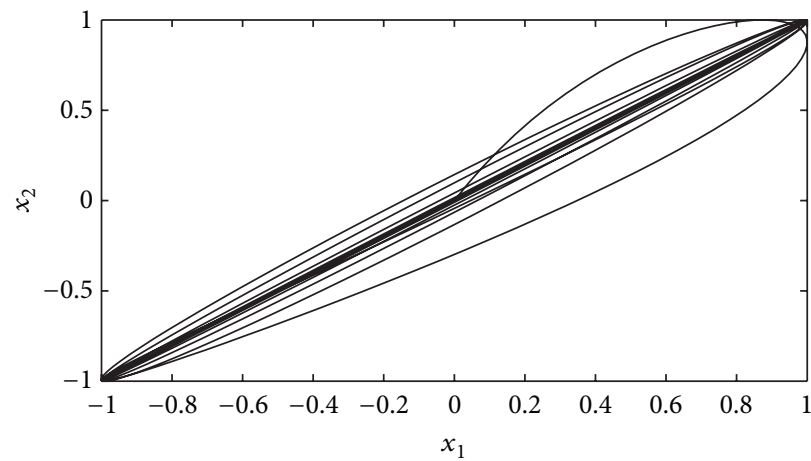

(a)

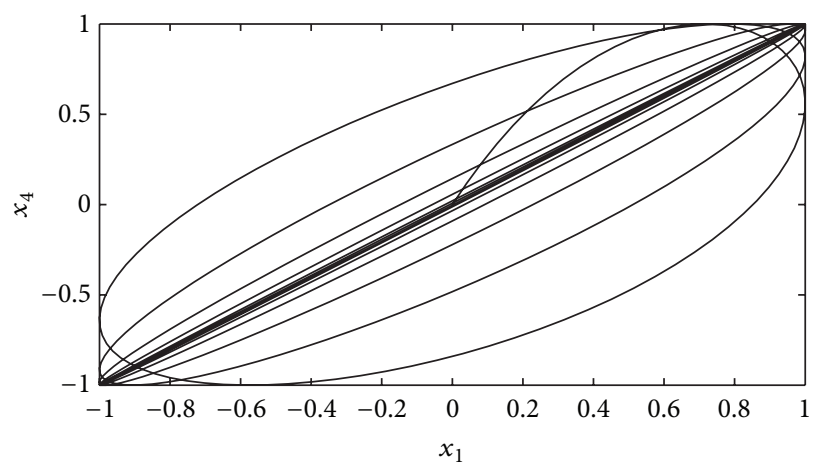

(c)

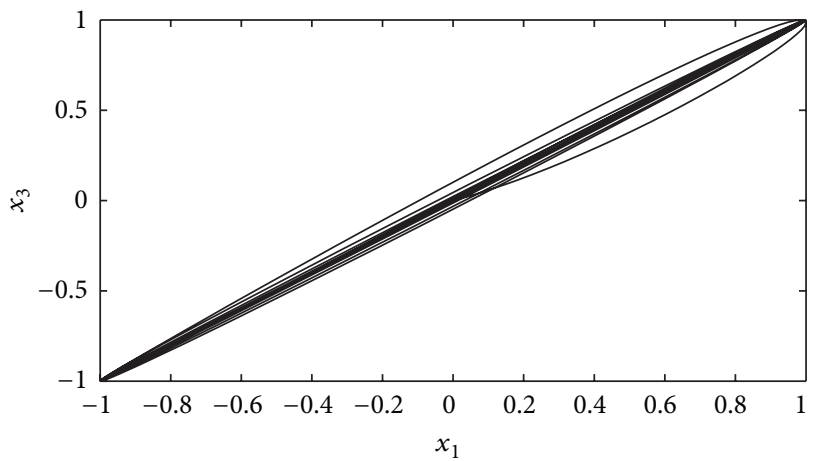

(b)

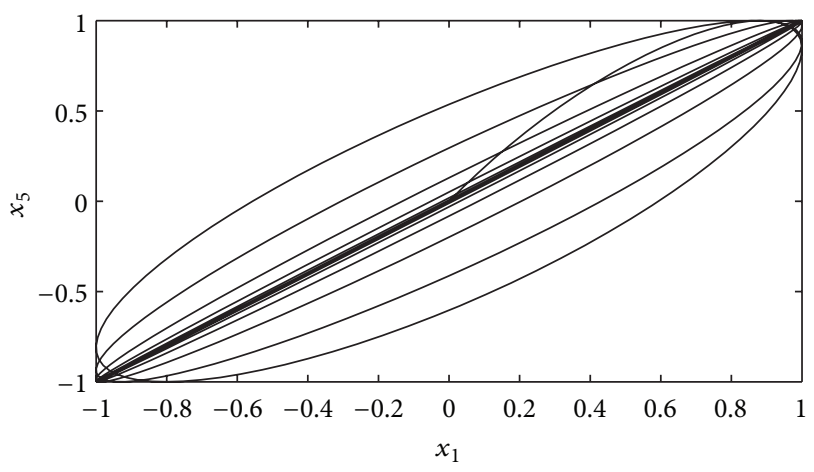

(d)

Figure 7: Phase diagrams (a) $x_{1}$ versus $x_{2}$, (b) $x_{1}$ versus $x_{3}$, (c) $x_{1}$ versus $x_{4}$, and (d) $x_{1}$ versus $x_{5}$ in the coupled nearest-neighbor network.

motion; see Figure 1, where the angular position $\theta$ is a point in the unitary circle and a short flash or pulse of the firefly occurs when a rotation is completed.

Figure 2 shows the behavior of isolated firefly (7) for initial conditions $x(0)=0, t(0)=0, f=2 \mathrm{~Hz}$, and $k=600$ iterations (note that, for appreciation purposes, we use the interpolation option of the Matlab simulation software).

\section{Network Synchronization of Coupled Fireflies in Different Connection Topologies}

In this section, network synchronization of coupled fireflies (7) in different topologies and the corresponding numerical results are reported. In the following cases of study, we assume that all the fireflies are connected, without self-loops and 


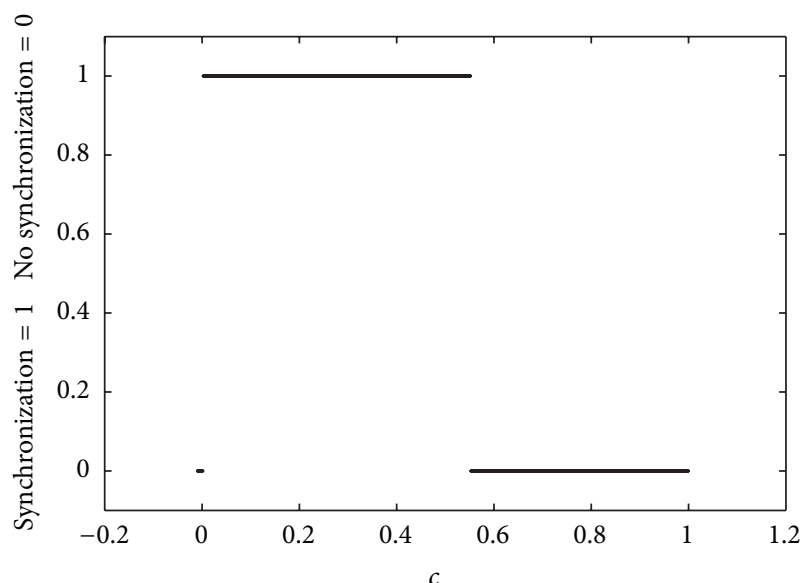

FIGURE 8: Synchronization diagram for the nearest-neighbor network.

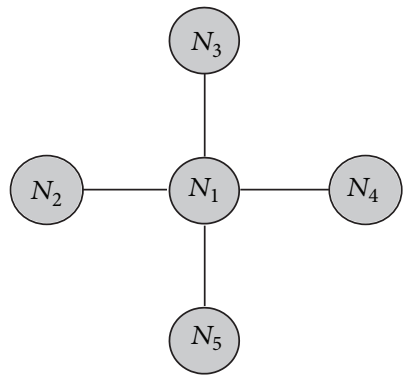

FIGURE 9: Graph for a star network corresponding to As.

without multiple edges between two fireflies. To be consistent with notation in complex network (1)-(2), we have considered the following change of variable:

$$
\mathbf{x}_{i}=\left[\begin{array}{l}
x_{i 1} \\
x_{i 2}
\end{array}\right]=\left[\begin{array}{l}
w_{i 1} \\
x_{i 2}
\end{array}\right]=\left[\begin{array}{l}
w_{i} \\
x_{i}
\end{array}\right] .
$$

4.1. Nearest-Neighbor Coupled Network. First, we consider a complex network according to (1)-(2) composed of $N=$ 5 coupled fireflies periodic oscillators (7) as fundamental nodes in nearest-neighbor coupled topology; see Figure 3. The corresponding state equations to this dynamical network are arranged as follows: the first firefly $N_{1}$ is given by

$$
\begin{aligned}
w_{1}(k+1) & =w_{1}(k)+u_{11}(k), \\
x_{1}(k+1) & =\sin \left(w_{1}(k) t(k)\right), \\
u_{11}(k) & =c\left(-2 w_{1}(k)+w_{2}(k)+w_{5}(k)\right),
\end{aligned}
$$

the second firefly $\mathrm{N}_{2}$ is given by

$$
\begin{aligned}
w_{2}(k+1) & =w_{2}(k)+u_{21}(k), \\
x_{2}(k+1) & =\sin \left(w_{2}(k) t(k)\right), \\
u_{21}(k) & =c\left(-2 w_{2}(k)+w_{1}(k)+w_{3}(k)\right),
\end{aligned}
$$

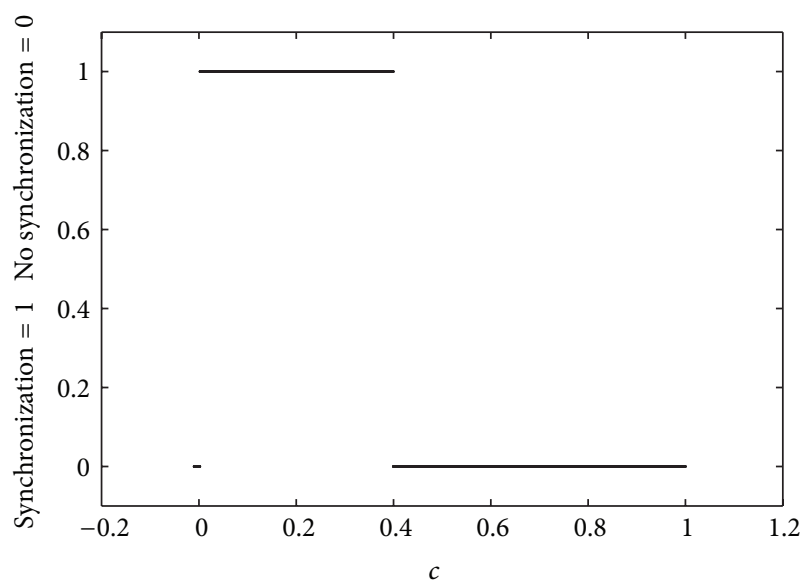

FIGURE 10: Synchronization diagram for the star network.

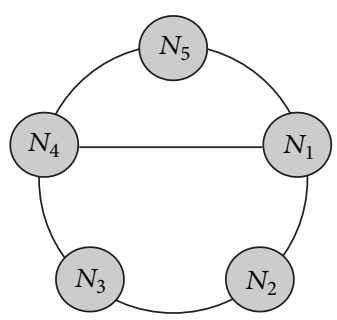

FIGURE 11: Graph for a small-world network corresponding to Asw.

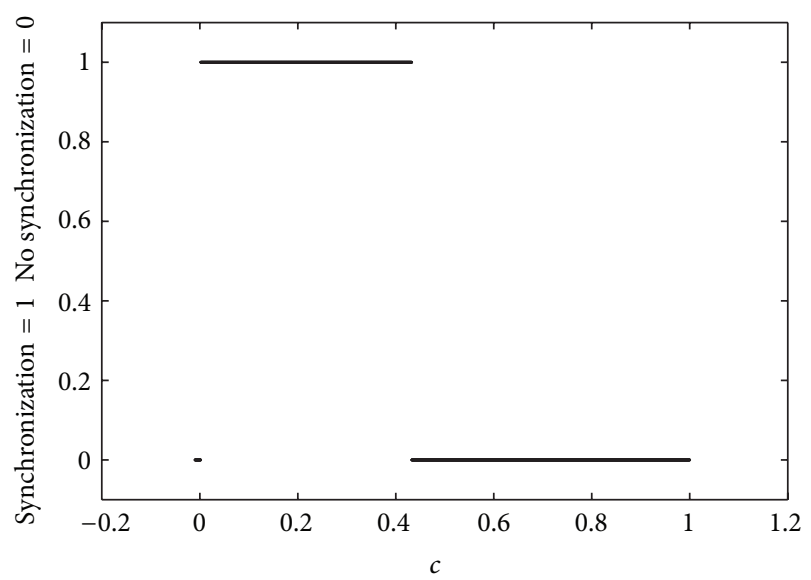

FIgURE 12: Synchronization diagram for the small-world network.

the third firefly $N_{3}$ is given by

$$
\begin{aligned}
w_{3}(k+1) & =w_{3}(k)+u_{31}(k), \\
x_{3}(k+1) & =\sin \left(w_{3}(k) t(k)\right), \\
u_{31}(k) & =c\left(-2 w_{3}(k)+w_{2}(k)+w_{4}(k)\right),
\end{aligned}
$$




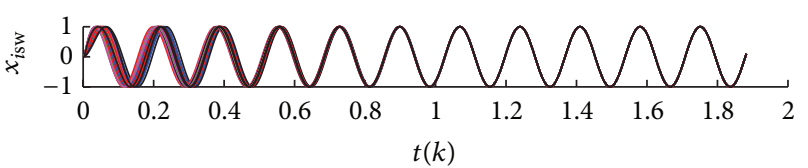

(a)

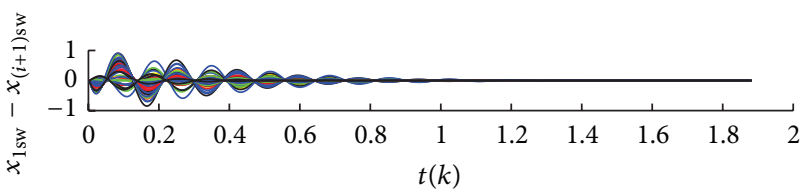

(c)

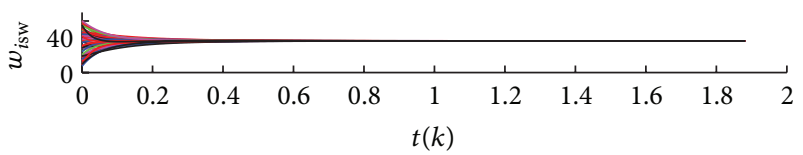

(b)

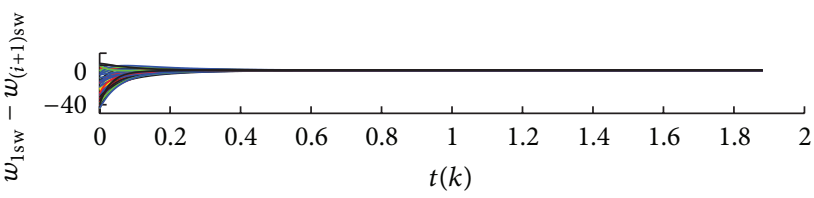

(d)

FIGURE 13: Small-world network in mass.

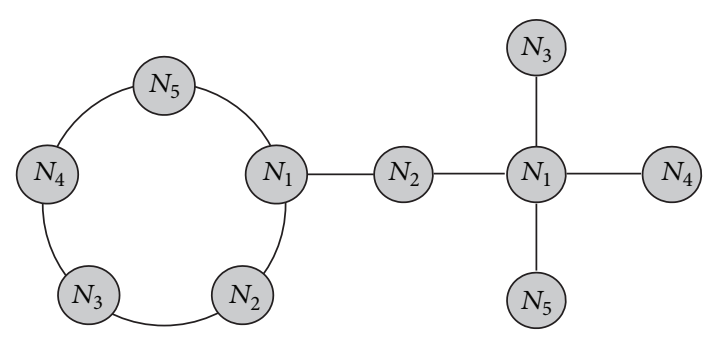

FIGURE 14: Graph of outer synchronization between two coupled complex networks, with different topologies.

the fourth firefly $N_{4}$ is given by

$$
\begin{aligned}
w_{4}(k+1) & =w_{4}(k)+u_{41}(k), \\
x_{4}(k+1) & =\sin \left(w_{4}(k) t(k)\right), \\
u_{41}(k) & =c\left(-2 w_{4}(k)+w_{3}(k)+w_{5}(k)\right),
\end{aligned}
$$

and the fifth firefly $N_{5}$ is given by

$$
\begin{aligned}
w_{5}(k+1) & =w_{5}(k)+u_{51}(k), \\
x_{5}(k+1) & =\sin \left(w_{5}(k) t(k)\right), \\
u_{51}(k) & =c\left(-2 w_{5}(k)+w_{4}(k)+w_{1}(k)\right),
\end{aligned}
$$

with

$$
t(k+1)=t(k)+\frac{\pi}{1000} .
$$

The corresponding coupling matrix A for the network shown in Figure 3 is given by

$$
A=\left[\begin{array}{ccccc}
-2 & 1 & 0 & 0 & 1 \\
1 & -2 & 1 & 0 & 0 \\
0 & 1 & -2 & 1 & 0 \\
0 & 0 & 1 & -2 & 1 \\
1 & 0 & 0 & 1 & -2
\end{array}\right]
$$

For illustrative purposes, we present the scenario where the oscillators fireflies of the network are uncoupled (isolated fireflies). From (9) to (28) by choosing $c=0$, the control inputs $u_{i 1}=0$; therefore, the five isolated fireflies of the network are unsynchronized assuming different initial conditions. Figures 4 and 5 show the collective behavior of five isolated fireflies oscillators: temporal dynamics of states $x_{i}(k)$ and $w_{i}(k)$ for $i=1,2, \ldots, 5$, synchronization errors $x_{1}(k)-$ $x_{i+1}(k)$ for $i=1,2,3,4$, and phase diagrams among the oscillators fireflies $x_{1}$ versus $x_{i}$ for $i=2,3,4,5$, with initial conditions $w_{1}(0)=2 \pi 2, w_{2}(0)=2 \pi 5, w_{3}(0)=2 \pi 1$, $w_{4}(0)=2 \pi 6, w_{5}(0)=2 \pi 4, x_{1}(0)=x_{2}(0)=x_{3}(0)=$ $x_{4}(0)=x_{5}(0)=0, t(0)=0$, and $k=600$. Consider now the scenario, where the oscillators fireflies of the network are coupled in nearest-neighbor topology (see Figure 3). For network synchronization purposes, we increase the coupling strength values of $c$, for $c>0$. Let $c=0.01$ with the same initial conditions as well as in uncoupled nearest-neighbor network. Figure 6 shows the temporal dynamics and errors, where $w_{1}(k)-w_{i+1}(k)$ is computed to observe the behavior of the error among the angular frequencies of the synchronized oscillators.

In Figure 7, we can appreciate the phase diagrams, where, after a transient, the involved signals overlap in a line of 45 degrees denoting synchronization. From Figures 6 and 7, we can deduce that, with this coupling strength and initial conditions, the network is synchronized. We obtained a range of coupling strength $c$ numerically. We compute (for $k=$ 5000 , so the calculation range of $c$ is approximate) a sweep of $c$ from 0 to 1 at intervals of 0.0001 . After removing the first 4000 iterations of each of the states, we compare the error between states $x_{i+1}(k)$ and $x_{1}(k)$ for $i=1,2,3,4$, where if the sum of absolute value of the errors is greater than 0.01 , then we established that the network is not synchronized (i.e., after 4000 iterations if $\left(\left|\left(x_{1}(k)-x_{2}(k)\right)\right|+\left|\left(x_{1}(k)-x_{3}(k)\right)\right|+\right.$ $\left.\left|\left(x_{1}(k)-x_{4}(k)\right)\right|+\left|\left(x_{1}(k)-x_{5}(k)\right)\right|\right)>0.01$, we established that there is no synchronization in the network). Figure 8 shows a diagram that we called synchronization diagram, where the value of 0 denotes no synchronization and the value of 1 denotes synchronization; thus, we can see the range $0.0019<c<0.5521$, where the nodes in the network are synchronized. 


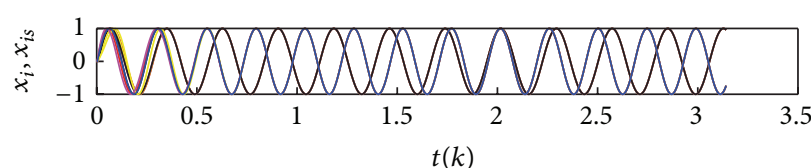

$-x_{i}$

$-x_{i s}$

(a)

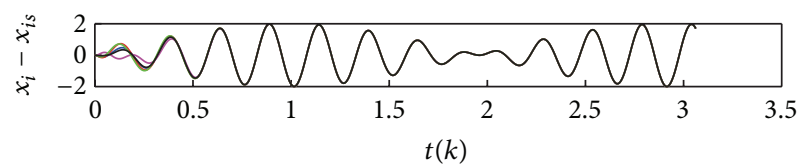

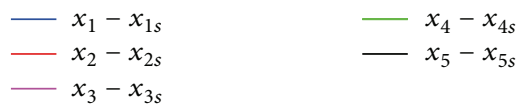

(c)

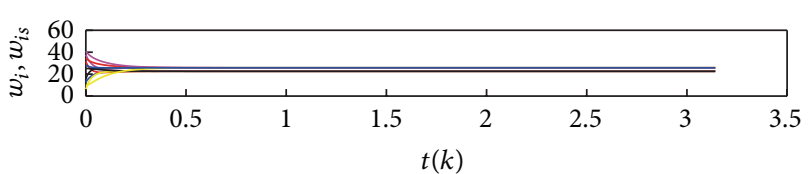

$-w_{i}$

(b)

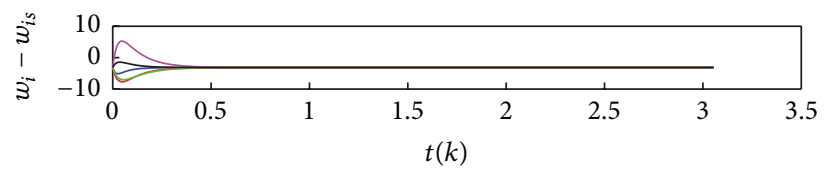

$\begin{array}{ll}- & w_{1}-w_{1 s} \\ - & w_{2}-w_{2 s} \\ - & w_{3}-w_{3 s}\end{array}$

$-w_{4}-w_{4 s}$

$-w_{5}-w_{5 s}$

(d)

FIGURE 15: Dynamics for two uncoupled networks with different topologies, temporal dynamics of (a) $x_{i}(k), x_{i s}(k),(\mathbf{b}) w_{i}(k), w_{i s}(k)$, and errors $(\mathrm{c}) x_{i}(k)-x_{i s}(k)$ and $(\mathrm{d}) w_{i}(k)-w_{i s}(k)$, where $i=1,2, \ldots, 5$.

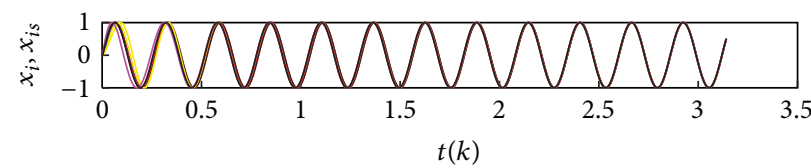

$x_{i}, x_{i s}$

(a)

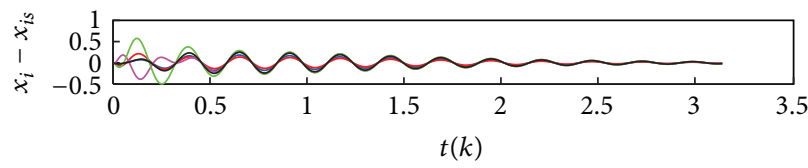

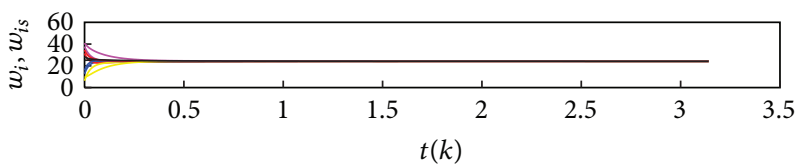

$-w_{i}, w_{i s}$

(b)

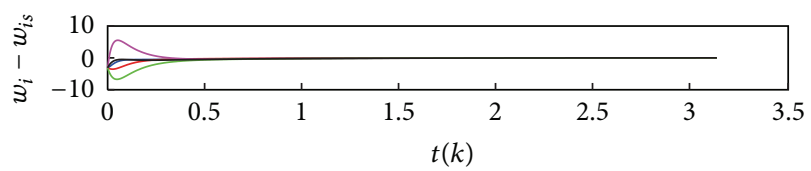

$$
-\begin{aligned}
& w_{1}-w_{1 s} \\
& w_{2}-w_{2 s}
\end{aligned}
$$$$
-w_{3}-w_{3 s}
$$

(c)

(d)

FIGURE 16: Dynamics for two coupled networks with different topologies, temporal dynamics of (a) $x_{i}(k), x_{i s}(k),(\mathrm{b}) w_{i}(k), w_{i s}(k)$, and errors (c) $x_{i}(k)-x_{i s}(k)$ and $(\mathrm{d}) w_{i}(k)-w_{i s}(k)$, where $i=1,2, \ldots, 5$.

4.2. Star Coupled Network. Now, we consider a complex network according to (1)-(2) composed of $N=5$ coupled fireflies periodic oscillators (7) as fundamental nodes in star coupled topology; see Figure 9. The corresponding state equations to this dynamical network are arranged as follows: the first firefly $N_{1}$ is given by

$$
\begin{aligned}
& w_{1 s}(k+1)=w_{1 s}(k)+u_{11 s}(k), \\
& x_{1 s}(k+1)=\sin \left(w_{1 s}(k) t(k)\right), \\
& u_{11 s}(k)=c\left(-4 w_{1 s}(k)+w_{2 s}(k)+\cdots+w_{3 s}(k)\right. \\
& \left.\quad+w_{4 s}(k)+w_{5 s}(k)\right),
\end{aligned}
$$

the second firefly $N_{2}$ is given by

$$
\begin{aligned}
w_{2 s}(k+1) & =w_{2 s}(k)+u_{21 s}(k), \\
x_{2 s}(k+1) & =\sin \left(w_{2 s}(k) t(k)\right), \\
u_{21 s}(k) & =c\left(-w_{2 s}(k)+w_{1 s}(k)\right),
\end{aligned}
$$

the third firefly $N_{3}$ is given by

$$
\begin{aligned}
w_{3 s}(k+1) & =w_{3 s}(k)+u_{31 s}(k), \\
x_{3 s}(k+1) & =\sin \left(w_{3 s}(k) t(k)\right), \\
u_{31 s}(k) & =c\left(-w_{3 s}(k)+w_{1 s}(k)\right),
\end{aligned}
$$




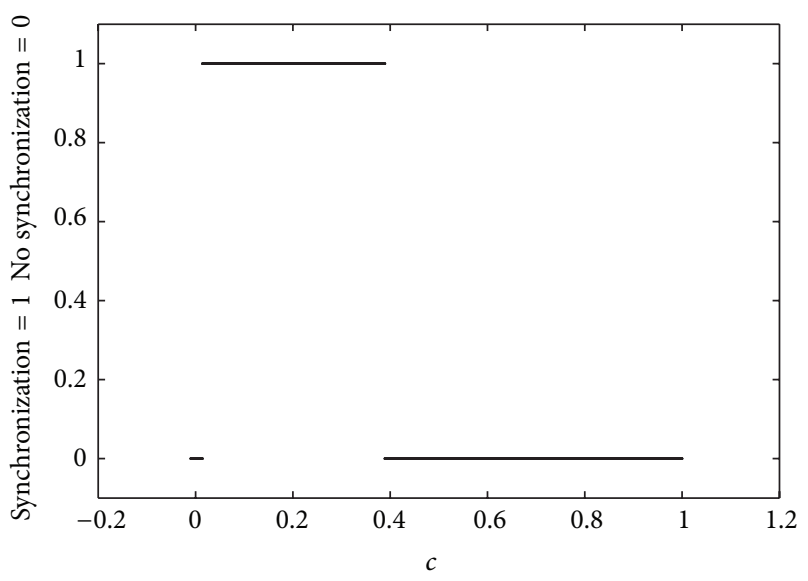

FIGURE 17: Synchronization diagram for outer synchronization of nearest-neighbor-star networks.

the fourth firefly $N_{4}$ is given by

$$
\begin{aligned}
w_{4 s}(k+1) & =w_{4 s}(k)+u_{41 s}(k), \\
x_{4 s}(k+1) & =\sin \left(w_{4 s}(k) t(k)\right), \\
u_{41 s}(k) & =c\left(-w_{4 s}(k)+w_{1 s}(k)\right),
\end{aligned}
$$

and the fifth firefly $N_{5}$ is given by

$$
\begin{aligned}
w_{5 s}(k+1) & =w_{5 s}(k)+u_{51 s}(k), \\
x_{5 s}(k+1) & =\sin \left(w_{5 s}(k) t(k)\right), \\
u_{51 s}(k) & =c\left(-w_{5 s}(k)+w_{1 s}(k)\right),
\end{aligned}
$$

with

$$
t(k+1)=t(k)+\frac{\pi}{1000} .
$$

The corresponding coupling matrix As for the network shown in Figure 9 is given by

$$
A s=\left[\begin{array}{ccccc}
-4 & 1 & 1 & 1 & 1 \\
1 & -1 & 0 & 0 & 0 \\
1 & 0 & -1 & 0 & 0 \\
1 & 0 & 0 & -1 & 0 \\
1 & 0 & 0 & 0 & -1
\end{array}\right],
$$

where subscript $s$ refers to the star network. We carry out the numerical simulations in the star network with $c=0.01$ and initial conditions $w_{1 s}(0)=2 \pi 2.5, w_{2 s}(0)=2 \pi 5.5, w_{3 s}(0)=$ $2 \pi 1.5, w_{4 s}(0)=2 \pi 6.5, w_{5 s}(0)=2 \pi 4.5, x_{1 s}(0)=x_{2 s}(0)=$ $x_{3 s}(0)=x_{4 s}(0)=x_{5 s}(0)=0, t(0)=0$, and $k=600$. Taking into account the above conditions, we obtain similar results as in the nearest-neighbor network and synchronization among the nodes was achieved. Figure 10 shows the synchronization diagram, where we can see the range $0.0021<c<0.3995$, where the nodes in the network are synchronized.
4.3. Small-World Coupled Network. A network with complex coupled topology can be represented by a random graph, but our intuition tells us clearly that many real complex networks are neither completely random nor completely regular. Something that we must emphasize is that most of the biological, technological, and social networks lie between these two extremes. These systems are highly clustered as regular arrays; however, they have a characteristic path length, like random networks; see [21]. These types of networks are called smallworld networks, in analogy to the small-world phenomenon (popularly known as six degrees of separation).

In general terms, these networks are regular arrangements made by connections or reconnections of pairs of randomly chosen nodes. Even a very small number of these added connections, commonly called shortcuts, do not change the local properties (very high clustering typical of a regular network) and cause those typical random networks values to be present in the average path length; see [22].

Now, we consider a complex network according to (1)(2) composed of $N=5$ coupled fireflies periodic oscillators (7) as fundamental nodes in small-world coupled topology; see Figure 11. The corresponding state equations to this dynamical network are arranged as follows: the first firefly $N_{1}$ is given by

$$
\begin{aligned}
& w_{1 \mathrm{sw}}(k+1)=w_{1 \mathrm{sw}}(k)+u_{11 \mathrm{sw}}(k), \\
& x_{1 \mathrm{sw}}(k+1)=\sin \left(w_{1 \mathrm{sw}}(k) t(k)\right), \\
& u_{11 \mathrm{sw}}(k)=c\left(-3 w_{1 \mathrm{sw}}(k)+w_{2 \mathrm{sw}}(k)+w_{4 \mathrm{sw}}(k)+\cdots\right. \\
& \left.\quad+w_{5 \mathrm{sw}}(k)\right),
\end{aligned}
$$

the second firefly $N_{2}$ is given by

$$
\begin{aligned}
w_{2 \mathrm{sw}}(k+1) & =w_{2 \mathrm{sw}}(k)+u_{21 \mathrm{sw}}(k), \\
x_{2 \mathrm{sw}}(k+1) & =\sin \left(w_{2 \mathrm{sw}}(k) t(k)\right), \\
u_{21 \mathrm{sw}}(k) & =c\left(-2 w_{2 \mathrm{sw}}(k)+w_{1 \mathrm{sw}}(k)+w_{3 \mathrm{sw}}(k)\right),
\end{aligned}
$$

the third firefly $N_{3}$ is given by

$$
\begin{aligned}
w_{3 \mathrm{sw}}(k+1) & =w_{3 \mathrm{sw}}(k)+u_{31 \mathrm{sw}}(k), \\
x_{3 \mathrm{sw}}(k+1) & =\sin \left(w_{3 \mathrm{sw}}(k) t(k)\right), \\
u_{31 \mathrm{sw}}(k) & =c\left(-2 w_{3 \mathrm{sw}}(k)+w_{2 \mathrm{sw}}(k)+w_{4 \mathrm{sw}}(k)\right),
\end{aligned}
$$

the fourth firefly $N_{4}$ is given by

$$
\begin{aligned}
& w_{4 \mathrm{sw}}(k+1)=w_{4 \mathrm{sw}}(k)+u_{41 \mathrm{sw}}(k), \\
& x_{4 \mathrm{sw}}(k+1)=\sin \left(w_{4 \mathrm{sw}}(k) t(k)\right), \\
& u_{41 \mathrm{sw}}(k)=c\left(-3 w_{4 \mathrm{sw}}(k)+w_{1 \mathrm{sw}}(k)+w_{3 \mathrm{sw}}(k)+\cdots\right. \\
& \left.\quad+w_{5 \mathrm{sw}}(k)\right),
\end{aligned}
$$

and the fifth firefly $N_{5}$ is given by

$$
\begin{aligned}
w_{5 \mathrm{sw}}(k+1) & =w_{5 \mathrm{sw}}(k)+u_{51 \mathrm{sw}}(k), \\
x_{5 \mathrm{sw}}(k+1) & =\sin \left(w_{5 \mathrm{sw}}(k) t(k)\right), \\
u_{51 \mathrm{sw}}(k) & =c\left(-2 w_{5 \mathrm{sw}}(k)+w_{4 \mathrm{sw}}(k)+w_{1 \mathrm{sw}}(k)\right),
\end{aligned}
$$




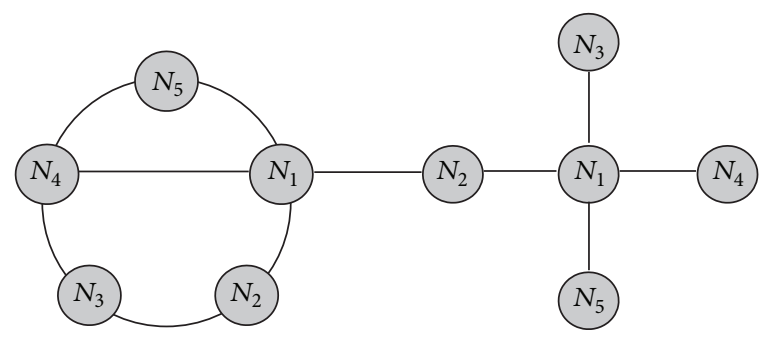

(a)

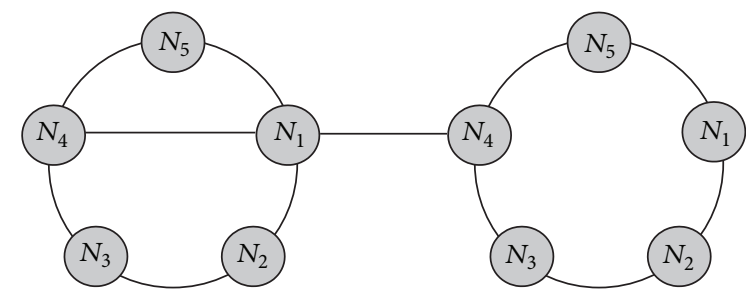

(c)

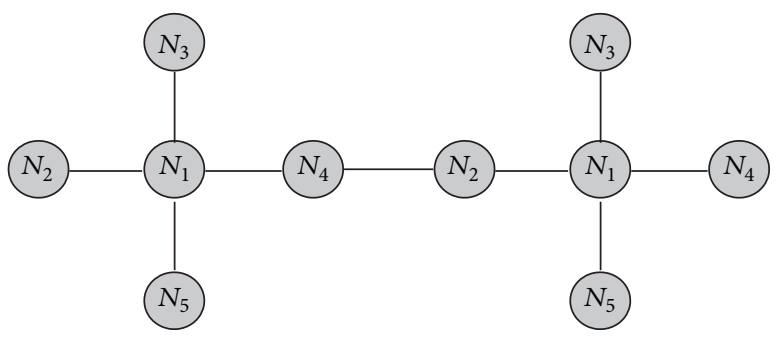

(b)

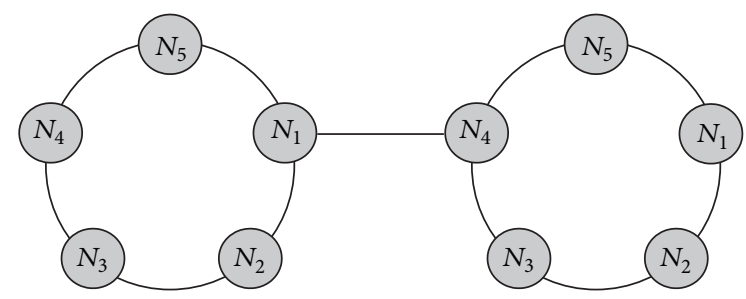

(d)

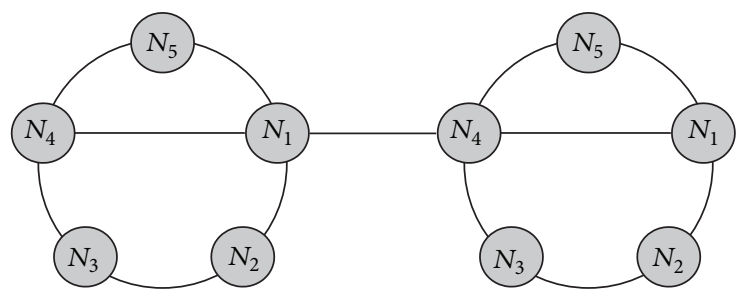

(e)

FIGURE 18: Cases for which outer synchronization was verified: (a) small-world-star, (b) star-star, (c) small-world-nearest-neighbor, (d) nearest-neighbor-nearest-neighbor, and (e) small-world-small-world.

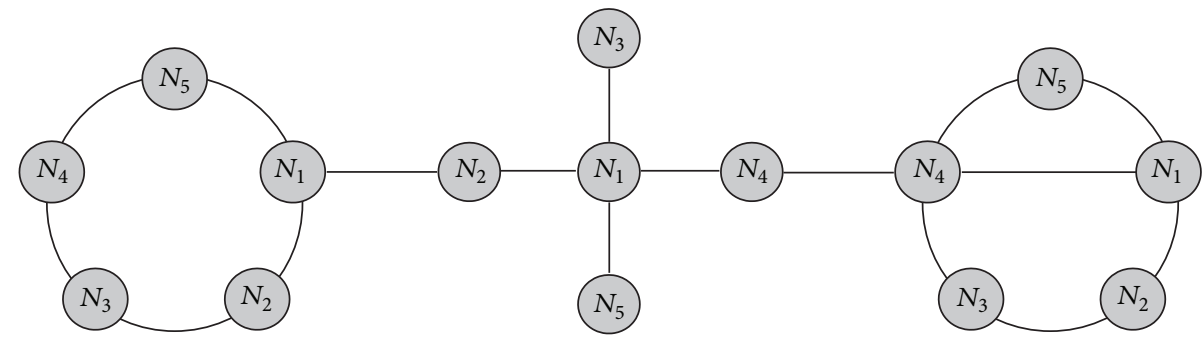

FIGURE 19: Graph of outer synchronization for three coupled networks.

with

$$
t(k+1)=t(k)+\frac{\pi}{1000} .
$$

The corresponding coupling matrix Asw for the network shown in Figure 11 is given by

$$
A s w=\left[\begin{array}{ccccc}
-3 & 1 & 0 & 1 & 1 \\
1 & -2 & 1 & 0 & 0 \\
0 & 1 & -2 & 1 & 0 \\
1 & 0 & 1 & -3 & 1 \\
1 & 0 & 0 & 1 & -2
\end{array}\right]
$$

where subscript sw refers to the small-world network.
We carry out the numerical simulations in the smallworld network with $c=0.01$ and initial conditions $w_{1 \mathrm{sw}}(0)=$ $2 \pi 2.3, w_{2 \mathrm{sw}}(0)=2 \pi 5.3, w_{3 \mathrm{sw}}(0)=2 \pi 1.3, w_{4 \mathrm{sw}}(0)=2 \pi 6.3$, $w_{5 \mathrm{sw}}(0)=2 \pi 4.3, x_{1 \mathrm{sw}}(0)=x_{2 \mathrm{sw}}(0)=x_{3 \mathrm{sw}}(0)=x_{4 \mathrm{sw}}(0)=$ $x_{5 \mathrm{sw}}(0)=0, t(0)=0$, and $k=600$. Taking into account the above conditions, we obtain similar results as in the nearestneighbor and star networks and synchronization among the nodes was achieved. Figure 12 shows the synchronization diagram, where we can see the range $0.0017<c<0.4325$, where the nodes in the network are synchronized.

With diffusive coupling, a network topology plays an important role in synchronization; see [23, 24]. We can corroborate this by analyzing and comparing the synchronization diagrams of the three topologies presented so far. 


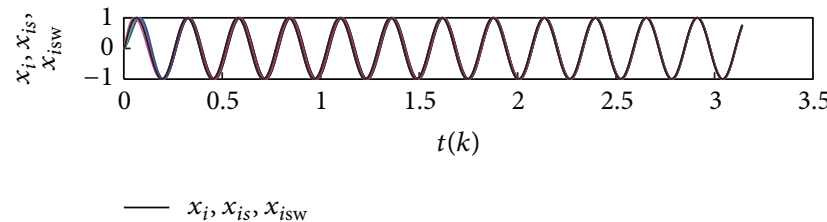

(a)

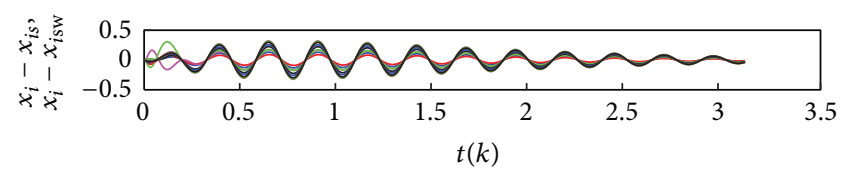

$$
\begin{array}{ll}
-x_{1}-x_{1 s}, x_{1}-x_{1 \mathrm{sw}} & -x_{4}-x_{4 s}, x_{4}-x_{4 \mathrm{sw}} \\
-x_{2}-x_{2 s}, x_{2}-x_{2 \mathrm{sw}} & x_{5}-x_{5 s}, x_{5}-x_{5 \mathrm{sw}} \\
-x_{3}-x_{3 s}, x_{3}-x_{3 \mathrm{sw}} &
\end{array}
$$

(c)

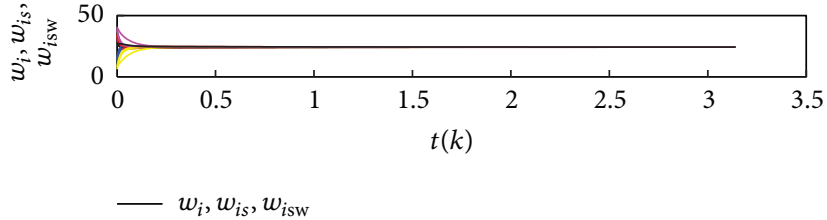

(b)

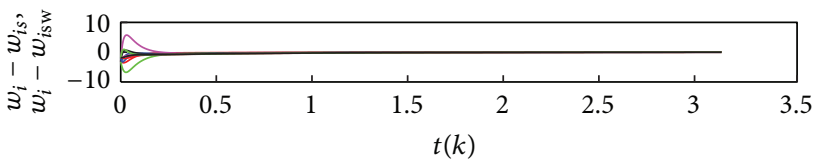

$-w_{1}-w_{1 s}, w_{1}-w_{1 \mathrm{sw}} \quad-w_{4}-w_{4 s}, w_{4}-w_{4 \mathrm{sw}}$
$-w_{2}-w_{2 s}, w_{2}-w_{2 \mathrm{sw}}$
$w_{3}-w_{3 s}, w_{3}-w_{3 \mathrm{sw}}$

(d)

Figure 20: Dynamics for three coupled networks with different topologies, temporal dynamics of (a) $x_{i}(k), x_{i s}(k)$, and $x_{i s w}(k)$ and (b) $w_{i}(k)$, $w_{i s}(k)$, and $w_{i s w}(k)$ and errors $(\mathrm{c}) x_{i}(k)-x_{i s}(k), x_{i}(k)-x_{i s w}(k)$ and $(\mathrm{d}) w_{i}(k)-w_{i s}(k), w_{i}(k)-w_{i s w}(k)$, where $i=1,2, \ldots, 5$.

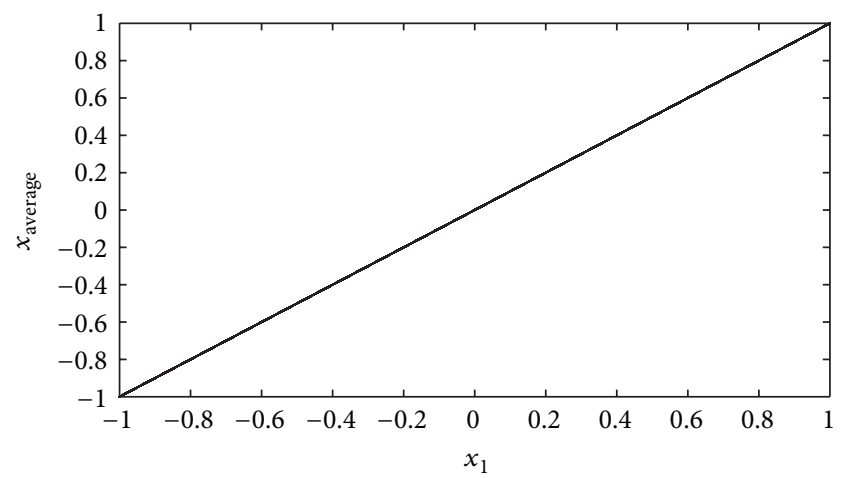

Figure 21: Phase diagram $\mathbf{x}_{\text {average }}$ versus $x_{1}$ for three coupled networks.

4.4. Small-World Coupled Network in Mass. Now, we use $N=$ 50 nodes in small-world network, with the only purpose to emulate the interaction of fireflies and its synchronization in mass.

Starting from a nearest-neighbor coupled topology, we add 100 connections with the same probability to build a small-world network, resulting in an average degree of $z=6$. For obvious reasons, we omit representations of the coupling matrix and graph on small-world network with $N=50$. Figure 13 shows the synchronization with the simple model proposed. Initial conditions are $x_{i s w}(0)=0$ and $w_{i s \mathrm{w}}(0)=2 \pi f_{i \mathrm{sw}}$, where $f_{\text {isw }}$ is randomly selected from 1 to $10 \mathrm{~Hz}$, with $i=1,2, \ldots, 50, t(0)=0, c=0.01$, and $k=600$. Figure 13 shows the simulation results, where synchronization is achieved for a small-world network with $N=50$ nodes.

\section{Outer Synchronization}

Outer synchronization occurs among coupled complex networks, which means that the corresponding nodes of the

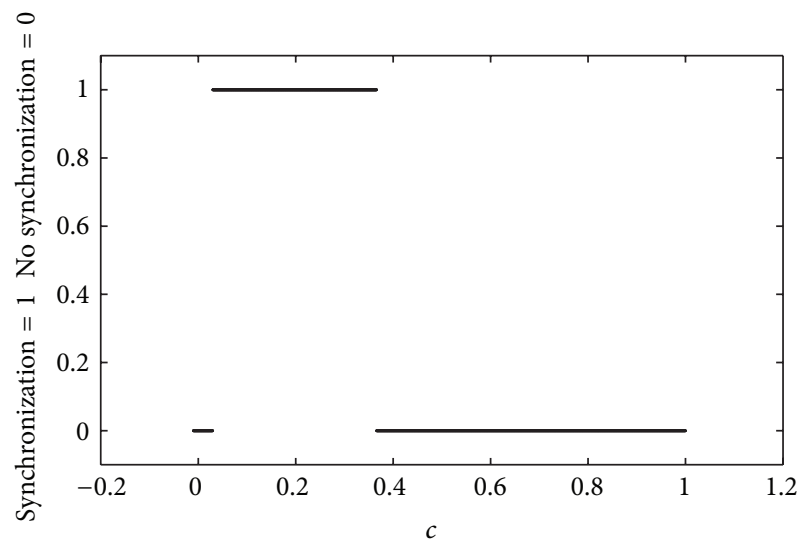

FIGURE 22: Synchronization diagram for outer synchronization of nearest-neighbor-star-small-world networks.

coupled networks will achieve synchronization [25-29]. The interaction among communities (or networks) is something that exists in our real world. One important example is the infectious disease that spreads among different communities. For example, avian influenza spreads among domestic and wild birds, afterward infecting human beings unexpectedly; AIDS, mad cow disease, bird flu, and SARS were originally spread between two communities (or networks). This means that the study of dynamics among coupled networks is necessary and important [30]. We use the complex network theory that was shown in Section 2 to couple a pair of nodes between two networks and obtain synchronization among the nodes of the networks (outer synchronization).

5.1. Outer Synchronization of Two Networks. Suppose two networks are the following: one in nearest-neighbor coupled topology and another in star coupled topology, and these networks are coupled as in Figure 14. First we show the case where the two networks are decoupled. Figure 15 shows two 


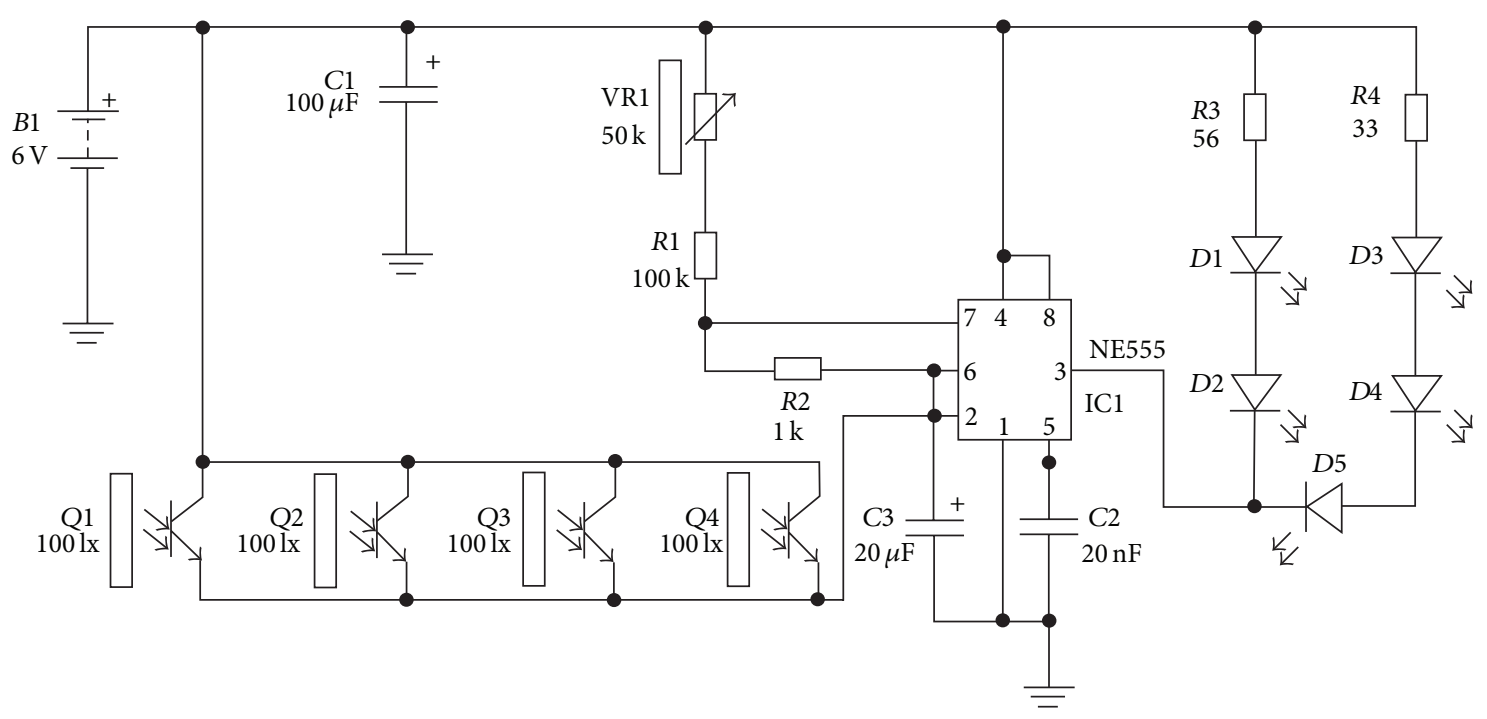

FIGURE 23: Electronic circuit of a simple firefly.

synchronization states belonging to each one of the networks; that is, the nodes of each network are synchronized, but the two networks are unsynchronized. Also, we can observe that the angular frequencies of the two networks tend to different values, so it is obvious that the two networks are not synchronized. If we couple the two networks, that is, if we connect $N_{1}$ of the nearest-neighbor network with $N_{2}$ of the star network, and we use a coupling strength $c=0.03$ with initial conditions as previously mentioned in Sections 4.1 and 4.2, outer synchronization is obtained as shown in Figure 16, where we clearly see that the dynamics of the states $x_{i}(k)$ and $x_{i s}(k)$ as well as the states $w_{i}(k)$ and $w_{i s}(k)$ tend to the same value, and errors among the states of the two networks will be zero. As expected, the synchronization time for both networks is greater than the synchronization time of a single network even with the increase of the coupling constant $c$, so in this case we simulate $k=1000$ iterations.

Figure 17 shows the synchronization diagram, where we can see the range $0.0142<c<0.3891$, where the nodes among the two networks are synchronized. We perform the numerical verification to the cases shown in Figure 18 with $c=0.03$ and initial conditions as previously mentioned in Sections 4.1, 4.2, and 4.3, achieving synchronization in each of the presented combinations.

5.2. Outer Synchronization of the Three Different Topologies. To complement the study of outer synchronization, we present the outer synchronization among the networks that have been presented in this paper.

Consider three networks (Figures 3, 9, and 11). We omit the case where networks are decoupled. Assume that the networks are coupled as in Figure 19. The corresponding results for the coupling among the three networks (Figure 19) are shown in Figure 20, where $c=0.05, k=1000$, and the initial conditions are those previously used in Sections 4.1, 4.2, and 4.3. We also include a phase diagram in Figure 21, where the abscissa axis is the average of the states $\mathbf{x}(k)$ and the ordinate axis is the state $x_{1}(k)$; we use $k=10000$ in order to eliminate the transient whereby outer synchronization can be verified. Figure 22 shows the synchronization diagram, where we can see the range $0.0297<c<0.3659$, where the nodes among the three networks are synchronized.

\section{Electronic Implementation}

Electronic firefly has been proposed in [31] to emulate the firefly flashing process. Nine circuits representing fireflies were built and synchronized in [32]. Here, we reproduce such circuit and we coupled nine fireflies in different topologies to realize the experimental fireflies synchronization. A firefly can be constructed with electronic components as 555 IC, resistances, capacitors, LEDs, IR, and phototransistors (Figure 23). The nine electronic fireflies are in a $3 \times 3$ array constructed in a $17 \times 19 \mathrm{~cm}$ phenolic plate (Figure $24(\mathrm{a})$ ). Experimental results are shown in Figure 24, where synchronization of coupled fireflies in different topologies is achieved. Synchronization was verified with the sensing pulses on a digital oscilloscope (Figure 25) [20]. The synchronization time is instantaneous because coupling strength is considered strong based on [32].

\section{Conclusions}

In this paper, network synchronization and outer synchronization problems with simple firefly discrete models have been investigated by using the theory of complex systems. The simulation results show the effective synchronization and outer synchronization with the simple model proposed. All the results presented help to understand how the collective behaviors work in nature and in living systems such as fireflies, crickets chirping, heart cell, neurons, and women's menstrual cycles. In addition, experimental network synchronization with nine electronic fireflies was presented. 


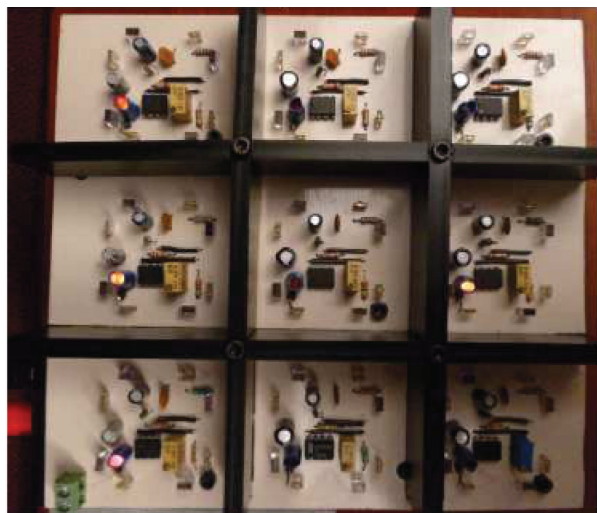

(a)

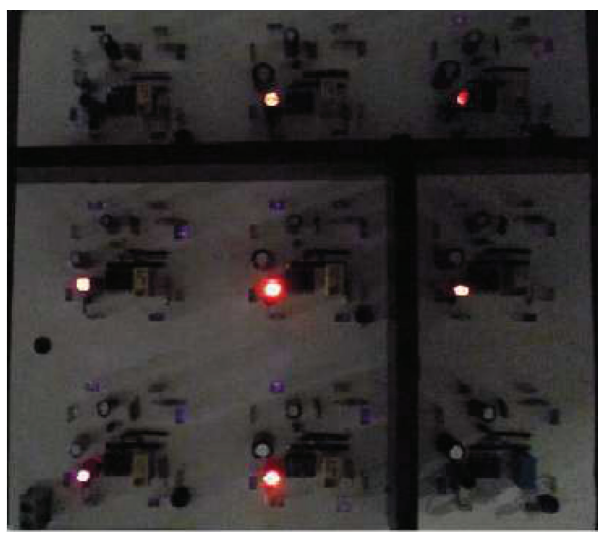

(c)

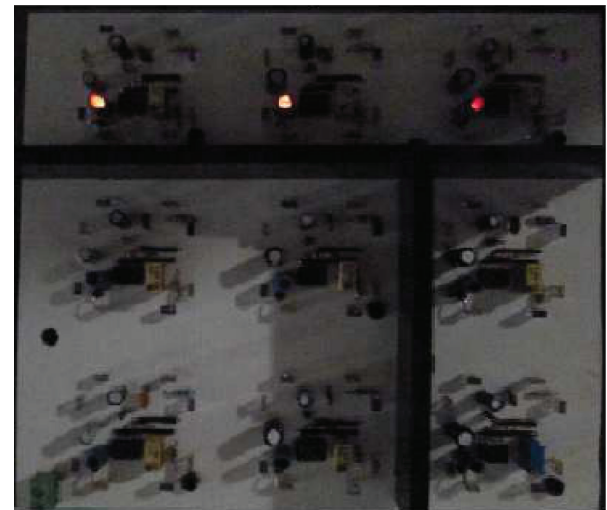

(b)

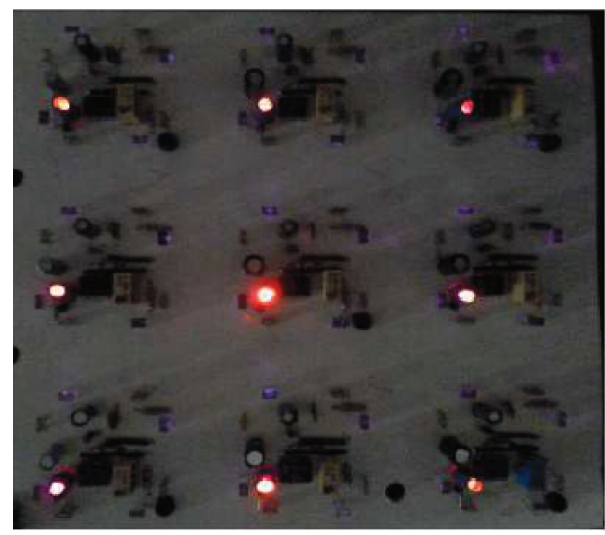

(d)

FIGURE 24: Experimental fireflies synchronization: (a) isolated nodes, (b) line network synchronization with $N=3$, (c) synchronization in three different networks with $N=4, N=3$, and $N=2$, and (d) network synchronization with $N=9$.

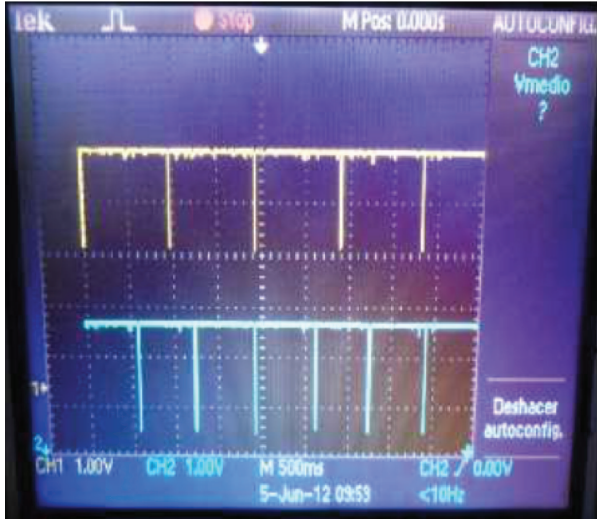

(a)

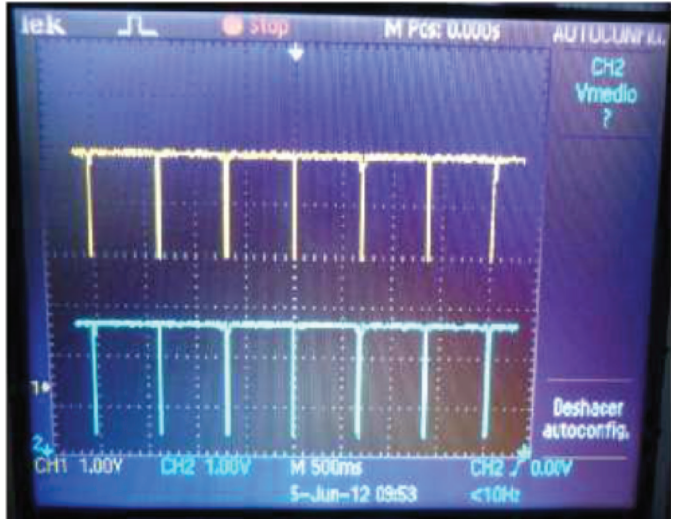

(b)

FIGURE 25: Synchronization verification between two fireflies by means of LED pulsation comparison by using an oscilloscope: (a) no synchronization and (b) synchronization.

\section{Conflict of Interests}

The authors declare that there is no conflict of interests regarding the publication of this paper.

\section{Acknowledgment}

This work was supported by the CONACYT, Mexico, under Research Grant 166654. 


\section{References}

[1] J. Buck, "Synchronous rhythmic flashing of fireflies. II," Quarterly Review of Biology, vol. 63, no. 3, pp. 265-289, 1988.

[2] H. M. Smith, "Synchronous flashing of fireflies," Science, vol. 82, no. 2120, pp. 151-152, 1935.

[3] M. Bennett, M. F. Schatz, H. Rockwood, and K. Wiesenfeld, "Huygens's clocks," The Royal Society of London. Proceedings, Series A: Mathematical, Physical and Engineering Sciences, vol. 458, no. 2019, pp. 563-579, 2002.

[4] A. Goldbeter, Biochemical Oscillations and Cellular Rhythms: The Molecular Bases of Periodic and Chaotic Behaviour, Cambridge University Press, 1996.

[5] C. Schäfer, M. G. Rosenblum, H. H. Abel, and J. Kurths, "Synchronization in the human cardiorespiratory system," Physical Review E, vol. 60, no. 1, pp. 857-870, 1999.

[6] B. Blasius, A. Huppert, and L. Stone, "Complex dynamics and phase synchronization in spatially extended ecological systems," Nature, vol. 399, no. 6734, pp. 354-359, 1999.

[7] K. M. Cuomo, A. V. Oppenheim, and S. H. Strogatz, "Synchronization of Lorenz-based chaotic circuits with applications to communications," IEEE Transactions on Circuits and Systems II: Analog and Digital Signal Processing, vol. 40, no. 10, pp. 626-633, 1993.

[8] S. H. Strogatz, SYNC: The Emerging Science of Spontaneous Order, Hyperion Press, New York, NY, USA, 2003.

[9] A. Pikovsky, M. Rosenblum, and J. Kurths, Synchronization: A Universal Concept in Nonlinear Sciences, Cambridge University Press, Cambridge, UK, 2001.

[10] A. T. Winfree, "Biological rhythms and the behavior of populations of coupled oscillators," Journal of Theoretical Biology, vol. 16, no. 1, pp. 15-42, 1967.

[11] C. S. Peskin, Mathematical Aspects of Heart Physiology, Courant Institute of Mathematical Sciences, 1975.

[12] J. Grasman and M. J. W. Jansen, "Mutually synchronized relaxation oscillators as prototypes of oscillating systems in biology," Journal of Mathematical Biology, vol. 7, no. 2, pp. 171197, 1979.

[13] T. Pavlidis, Biological Oscillators: Their Mathematical Analysis, Academic Press, 1973.

[14] R. E. Mirollo and S. H. Strogatz, "Synchronization of pulsecoupled biological oscillators," SIAM Journal on Applied Mathematics, vol. 50, no. 6, pp. 1645-1662, 1990.

[15] U. Ernst, K. Pawelzik, and T. Geisel, "Delay-induced multistable synchronization of biological oscillators," Physical Review E, vol. 57, no. 2, pp. 2150-2162, 1998.

[16] S. H. Strogatz, R. E. Mirollo, and P. Matthews, "Coupled nonlinear oscillators below the synchronization threshold: relaxation by generalized Landau damping," Physical Review Letters, vol. 68, no. 18, pp. 2730-2733, 1992.

[17] B. Ermentrout, "An adaptive model for synchrony in the firefly Pteroptyx malaccae," Journal of Mathematical Biology, vol. 29, no. 6, pp. 571-585, 1991.

[18] X. F. Wang and G. Chen, "Synchronization in small-world dynamical networks," International Journal of Bifurcation and Chaos in Applied Sciences and Engineering, vol. 12, no. 1, pp. 187192, 2002.

[19] X. F. Wang, "Complex networks: topology, dynamics and synchronization," International Journal of Bifurcation and Chaos in Applied Sciences and Engineering, vol. 12, no. 5, pp. 885-916, 2002.
[20] M. A. Murillo-Escobar, R. M. López-Gutiérrez, C. CruzHernández, and F. Abundiz-Pérez, Sincronizacion de Nueve Luciernagas Electronicas. XX Engineering, Architecture and Design Days FIAD-UABC, 2013.

[21] M. Newman, A.-L. Barabüsi, and D. J. Watts, Eds., The Structure and Dynamics of Networks, Princeton Studies in Complexity, Princeton University Press, Princeton, NJ, USA, 2006.

[22] N. Dorogovtsev and J. F. F. Mendes, Evolution of Networks: From Biological Nets to the Internet and $W W W$, Oxford University Press, Oxford, UK, 2003.

[23] R. C. G. Murguia, R. H. B. Fey, and H. Nijmeijer, "Network synchronization of time-delayed coupled nonlinear systems using predictor-based diffusive dynamic couplings," Chaos, vol. 25, no. 2, Article ID 023108, 2015.

[24] E. Steur, W. Michiels, H. Huijberts, and H. Nijmeijer, "Networks of diffusively time-delay coupled systems: conditions for synchronization and its relation to the network topology," Physica D: Nonlinear Phenomena, vol. 277, pp. 22-39, 2014.

[25] Z. Li and X. Xue, "Outer synchronization of coupled networks using arbitrary coupling strength," Chaos, vol. 20, no. 2, Article ID 023106, 7 pages, 2010.

[26] J. Feng, S. Sheng, Z. Tang, and Y. Zhao, "Outer synchronization of complex networks with nondelayed and time-varying delayed couplings via pinning control or impulsive control," Abstract and Applied Analysis, vol. 2015, Article ID 414596, 11 pages, 2015.

[27] X. Fang, Q. Yang, and W. Yan, “Outer synchronization between complex networks with nonlinear time-delay characteristics and time-varying topological structures," Mathematical Problems in Engineering, vol. 2014, Article ID 437673, 10 pages, 2014.

[28] S. Zheng, "Adaptive impulsive observer for outer synchronization of delayed complex dynamical networks with output coupling," Journal of Applied Mathematics, vol. 2014, Article ID 450193, 11 pages, 2014.

[29] D. Ning, X. Wu, J.-a. Lu, and H. Feng, "Generalized outer synchronization between complex networks with unknown parameters," Abstract and Applied Analysis, vol. 2013, Article ID 802859, 9 pages, 2013.

[30] C. Li, C. Xu, W. Sun, J. Xu, and J. Kurths, “Outer synchronization of coupled discrete-time networks," Chaos, vol. 19, no. 1, Article ID 013106, 2009.

[31] W. Garver and F. Moss, "Electronic fireflies," Scientific American, vol. 269, no. 6, pp. 128-130, 1993.

[32] T.-H. Chuang, C.-Y. Chang, J. Byerly, and C. Slattery, Synchronized Electronic Fireflies, 2014, http://www2.ece.ohio-state.edu/ $\sim$ passino/fireflyfolder/firefly.html. 


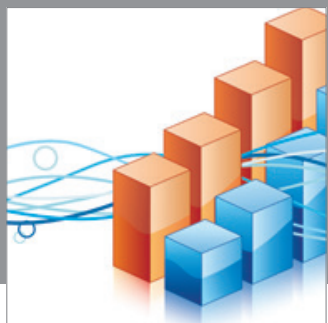

Advances in

Operations Research

mansans

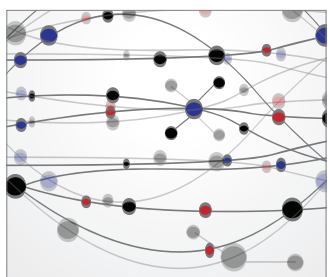

The Scientific World Journal
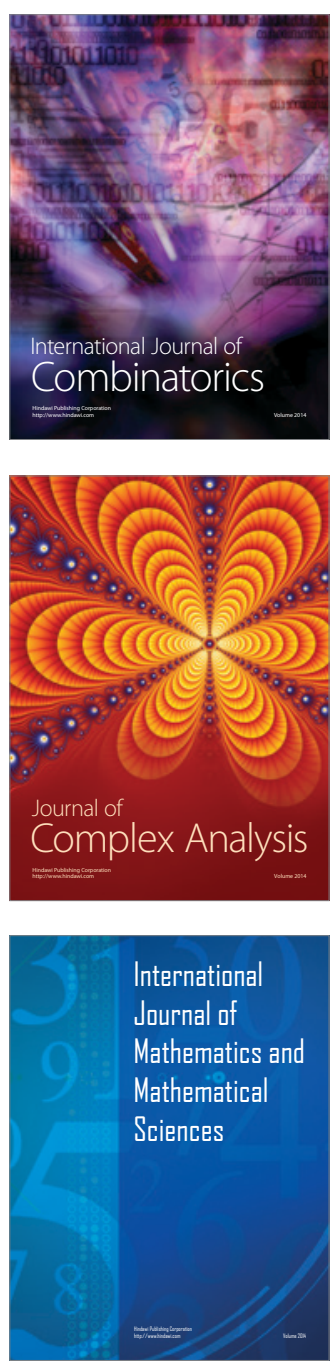
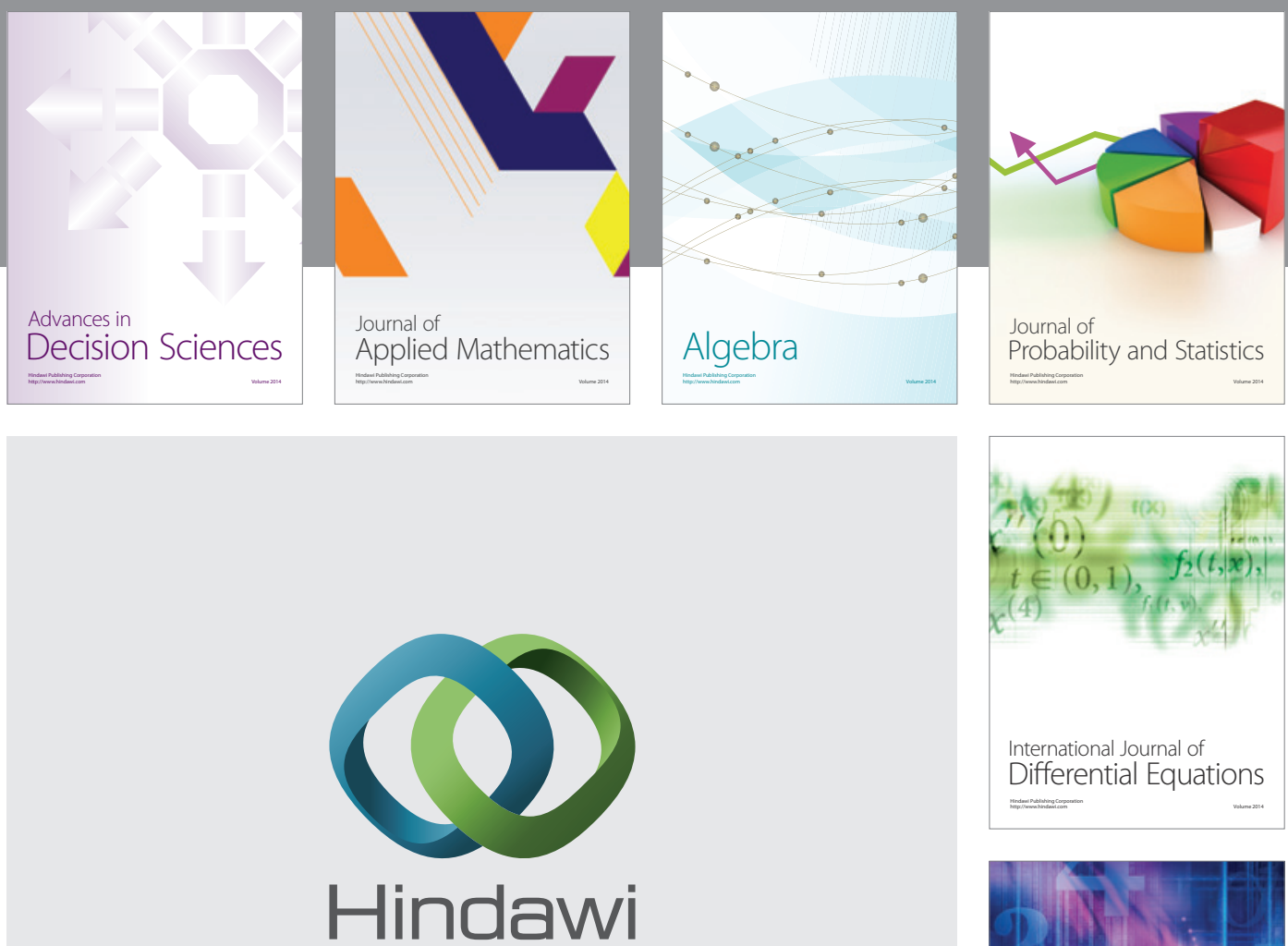

Submit your manuscripts at http://www.hindawi.com
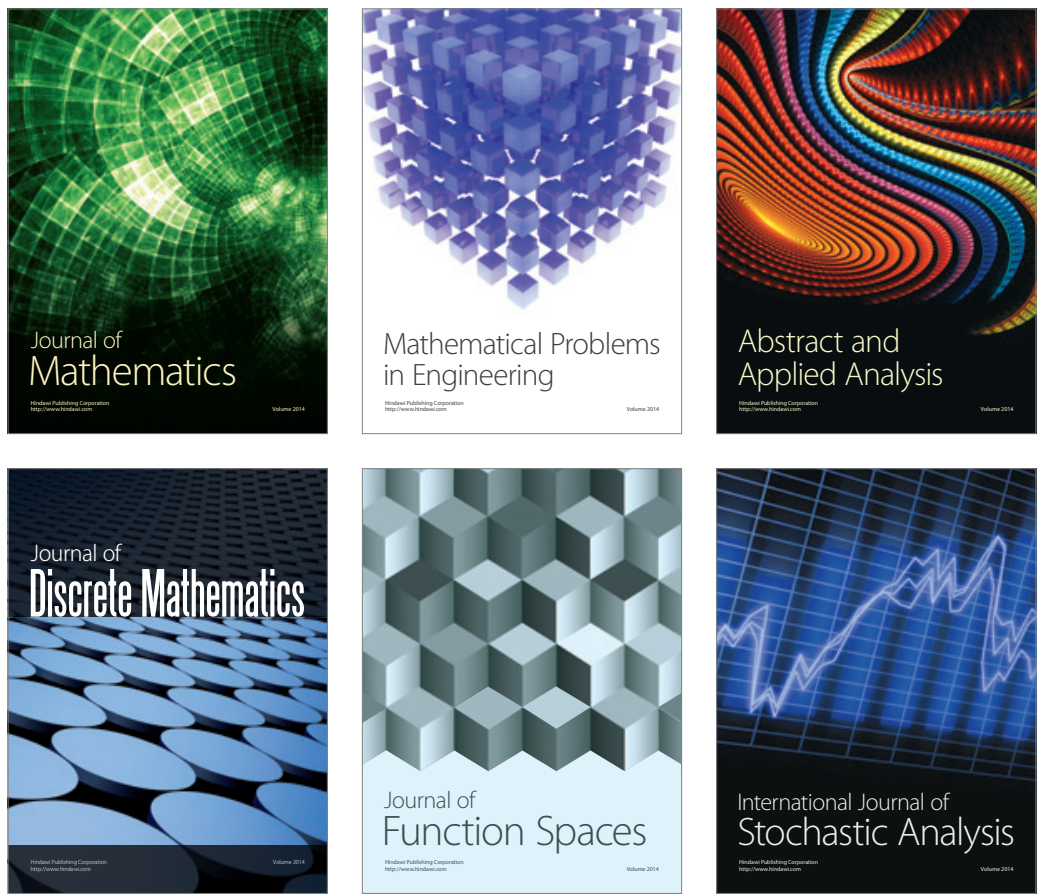

Journal of

Function Spaces

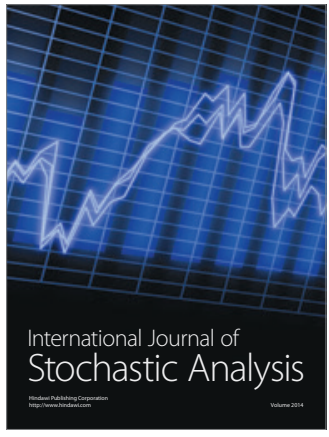

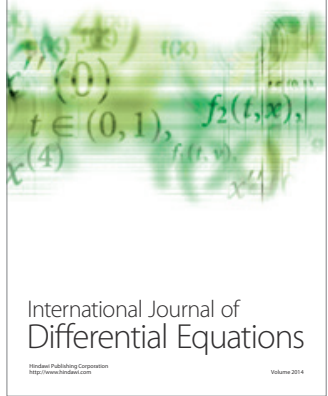
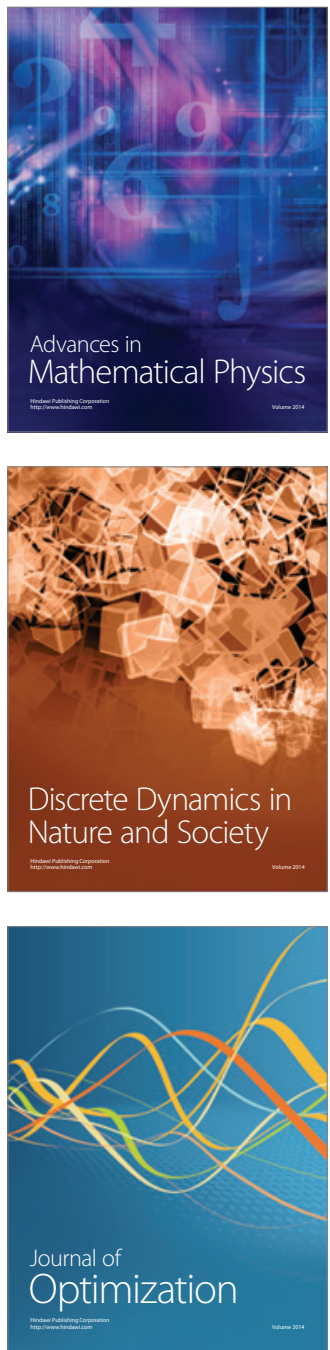